\title{
Theoretical investigation of noncollinear phase-matched parametric four-photon amplification of ultrashort light pulses in isotropic media
}

\author{
A. PENZKOFER, H. J. LEHMEIER \\ Naturwissenschaftliche Fakultät II - Physik, Universität Regensburg, D-93040 \\ Regensburg, Germany
}

Received 29 March; revised 23 June; accepted 1 July 1993

\begin{abstract}
The amplification of light signals (angular frequency $\omega_{\mathrm{S}}$ ) in some isotropic media $\left(\mathrm{D}_{2} \mathrm{O}\right.$, fused silica, and Schott type SF10 glasses) by noncollinear phase-matched parametric four-photon interaction $\omega_{1}+\omega_{2} \rightarrow \omega_{S}+\omega_{1}$ is studied theoretically. Computer simulations are carried out for fundamental and second-harmonic pump pulses of a mode-locked $\mathrm{Nd}$ :glass laser. Degenerate interaction (wavelength $\lambda_{1}=\lambda_{2}=1054 \mathrm{~nm}$ or $\left.527 \mathrm{~nm}\right)$ and nondegenerate interaction $\left(\lambda_{1}=1054 \mathrm{~nm}\right.$, $\lambda_{2}=527 \mathrm{~nm}$ ) are considered. Characteristic phase-matching parameters and gain parameters versus wavelength are determined. Limitations by spectral bandwidth, optical absorption, optical damage, self-phase modulation, self-focusing and stimulated Raman scattering are analysed.
\end{abstract}

\section{Introduction}

Parametric three-photon interaction in noncentrosymmetric crystals is widely used in parametric oscillators (feedback of generated light), generators (build-up from quantum noise and black-body radiation), and amplifiers (amplification of input signal) [1-5]. Parametric four-photon interaction is possible in all media [1-10]. Parametric four-photon generation (also called stimulated four-photon mixing $[10,11]$, stimulated parametric fourphoton interaction [12], or stimulated four-wave parametric emission [3]) plays an important role in the generation of picosecond light continua [3, 10-15 and references therein]. Four-photon parametric oscillators have been realized by applying optical fibres [16] and atomic vapours [6,17-21]. Also, parametric four-photon amplification has been achieved in optical fibres [10, 22-26] and atomic vapours [27-30]. In atomic vapours the resonant enhancement of the third-order nonlinear susceptibility near transition frequencies is exploited and phase-matching is achieved by the refractive index dispersion around optical transitions and by buffer gas addition [6]. In optical fibres high pump pulse intensities are maintained over long distances. Phase matching is achieved in certain wavelength regions by waveguide contributions to the refractive index in multimode optical fibres, and by modal birefringence in polarization-preserving single-mode fibres [10]. Theoretical studies of parametric four-photon amplification are presented in [13, 31-34]. 
In this paper the feasibility of parametric four-photon amplification of light pulses in bulk condensed isotropic media is investigated. Numerical simulations are performed for noncollinear phase-matched parametric four-photon amplification in heavy water, fused silica, and a flint glass of type Schott SF10. Degenerate interaction, $\omega_{1}+\omega_{1} \rightarrow \omega_{\mathrm{S}}+\omega_{\mathrm{I}}$ (monochromatic pumping; $\omega_{1}, \omega_{\mathrm{S}}$ and $\omega_{\mathrm{I}}$ are the angular frequencies of the pump, signal and idler pulse, respectively) and nondegenerate interaction, $\omega_{1}+\omega_{2} \rightarrow \omega_{\mathrm{S}}+\omega_{\mathrm{I}}$ with $\omega_{1} \neq \omega_{2}$ (bichromatic pumping) are considered. Calculations are performed for pump wavelengths $1054 \mathrm{~nm}$ and $527 \mathrm{~nm}$ of a mode-locked Nd:glass laser (fundamental and second-harmonic wavelength). Phase-matching angles are calculated, and spatial pulse overlap lengths and temporal spreadings of the pulses are determined. Parametric amplification factors are estimated. Limitation by spectral pulse bandwidth, optical absorption, optical damage, self-phase modulation, self-focusing and stimulated Raman scattering are analysed. Wavelength regions of allowed parametric four-photon amplification (positive gain) versus pump pulse duration are determined.

\section{Fundamentals}

Noncollinear phase-matched parametric four-photon amplification $\omega_{1}+\omega_{2} \rightarrow \omega_{\mathrm{S}}+\omega_{\mathrm{I}}$ is considered. Pump pulses at angular frequencies $\omega_{1}$ and $\omega_{2}$ (degenerate case: $\omega_{1}=\omega_{2}$; nondegenerate case: $\omega_{1} \neq \omega_{2}$ ) amplify an input signal at frequency $\omega_{\mathrm{S}}$ and thereby generate an idler signal at frequency $\omega_{\mathrm{I}}$ (no idler input signal). The pump pulses and the signal pulse are set to be non-divergent and linearly polarized in the same direction. Only the small-signal approximation limit is considered where the pump pulse intensities remain constant. Neglecting optical absorption, the collinear parametric four-photon amplification in the slowly varying amplitude approximation is described by Equations 1 and 2 $[1,10,12,14,15]$ :

$$
\begin{aligned}
& \frac{\partial E_{\mathrm{S}}}{\partial z}=-\mathrm{i} \frac{\omega_{\mathrm{S}}}{2 n_{\mathrm{S}} c_{0}} D\left(\omega_{1}, \omega_{2}\right) \chi_{x x x x}^{(3)}\left(-\omega_{\mathrm{S}} ; \omega_{1}, \omega_{2},-\omega_{\mathrm{I}}\right) E_{1} E_{2} E_{\mathrm{I}}^{*} \exp \left(\mathrm{i} \Delta k_{z} z\right) \\
& \frac{\partial E_{\mathrm{I}}}{\partial z}=-\mathrm{i} \frac{\omega_{\mathrm{I}}}{2 n_{\mathrm{I}} c_{0}} D\left(\omega_{1}, \omega_{2}\right) \chi_{x x x x}^{(3)}\left(-\omega_{\mathrm{I}} ; \omega_{1}, \omega_{2},-\omega_{\mathrm{S}}\right) E_{1} E_{2} E_{\mathrm{S}}^{*} \exp \left(\mathrm{i} \Delta k_{z} z\right)
\end{aligned}
$$

where $E_{\mathrm{S}}, E_{\mathrm{I}}, E_{1}$ and $E_{2}$ are the amplitudes of the electrical field strengths at the angular frequencies $\omega_{\mathrm{S}}$ (signal), $\omega_{\mathrm{I}}$ (idler), $\omega_{1}$ and $\omega_{2}$ (pump pulses); $c_{0}$ is the light velocity in vacuum; $z$ is the propagation direction; and $\Delta k_{z}=k_{\mathrm{S}, z}+k_{\mathrm{I}, z}-k_{1, z}-k_{2, z}=k_{\mathrm{S}}+$ $k_{\mathrm{I}}-k_{1}-k_{2}$ is the wavevector mismatch. The wavevectors $k_{i}(i=1,2, \mathrm{~S}, \mathrm{I})$ are given by $k_{i}=n_{i} \omega_{i} / c_{0}=2 \pi n_{i} \nu_{i} / c_{0}=2 \pi n_{i} \tilde{\nu}_{i}=2 \pi n_{i} / \lambda_{i}$, where the $n_{i}$ are the refractive indices at the angular frequencies $\omega_{i}$, frequencies $\nu_{i}$, wavenumbers $\tilde{\nu}_{i}$ or wavelengths $\lambda_{i}$. $\chi_{x x x x}^{(3)}\left(-\omega_{\mathrm{S}} ; \omega_{1}, \omega_{2},-\omega_{\mathrm{I}}\right)$ and $\chi_{x x x x}^{(3)}\left(-\omega_{\mathrm{I}} ; \omega_{1}, \omega_{2},-\omega_{\mathrm{S}}\right)$ are the third-order nonlinear optical susceptibility components. They are related by $[1,12] \chi_{x x x x}^{(3)}\left(-\omega_{\mathrm{S}} ; \omega_{1}, \omega_{2},-\omega_{\mathrm{I}}\right)=\chi_{x x x x}^{(3)^{*}}$ $\left(-\omega_{\mathrm{I}} ; \omega_{1}, \omega_{2},-\omega_{\mathrm{S}}\right)$, and we abbreviate $\chi_{x x x x}^{(3)}\left(-\omega_{\mathrm{S}} ; \omega_{1}, \omega_{2},-\omega_{\mathrm{I}}\right)$ by $\chi_{\mathbf{P}}^{(3)} . D\left(\omega_{1}, \omega_{2}\right)$ is the degeneracy factor of the nonlinear susceptibilities, where $D\left(\omega_{1}, \omega_{2}\right)=3$ for $\omega_{1}=\omega_{2}$ (degenerate or monochromatic case) and $D\left(\omega_{1}, \omega_{2}\right)=6$ for $\omega_{1} \neq \omega_{2}$ (nondegenerate or bichromatic case) $[1,35]$. The nonlinear susceptibilities are defined by the nonlinear polarization relation $\boldsymbol{P}_{\mathrm{NL}}=4 \epsilon_{0} \chi^{(3)}: \boldsymbol{E E E}$ [36] where $\epsilon_{0}$ is the permittivity of free space. 
The solution of the equation system $(1,2)$ in the parametric amplification limit (neglection of quantum noise amplification) is $[1,10,12]$

$$
\begin{gathered}
E_{\mathrm{S}}(z)=E_{\mathrm{S}}(0) \exp \left(\mathrm{i} \frac{\Delta k_{z} z}{2}\right)\left[\cosh (\beta z)-\mathrm{i} \frac{\Delta k_{z}}{2 \beta} \sinh (\beta z)\right] \\
E_{\mathrm{I}}^{*}(z)=\mathrm{i} \frac{\kappa_{\mathrm{I}}^{*}}{\beta} E_{\mathrm{S}}(0) \exp \left(-\mathrm{i} \frac{\Delta k_{z} z}{2}\right) \sinh (\beta z)
\end{gathered}
$$

where

$$
\beta=\left(\kappa_{\mathrm{S}}^{*} \kappa_{\mathrm{I}}-\Delta k_{z}^{2}\right)^{1 / 2}
$$

with

$$
\kappa_{\mathrm{I}}=\frac{\omega_{\mathrm{I}}}{2 n_{\mathrm{I}} c_{0}} D\left(\omega_{1}, \omega_{2}\right) \chi_{\mathrm{P}}^{(3)^{*}} E_{1} E_{2}
$$

and

$$
\kappa_{\mathrm{S}}=\frac{\omega_{\mathrm{S}}}{2 n_{\mathrm{S}} c_{0}} D\left(\omega_{1}, \omega_{2}\right) \chi_{\mathrm{P}}^{(3)} E_{1} E_{2}
$$

The pump pulse, signal, and idler intensities $I_{i}(i=1,2, \mathrm{~S}, \mathrm{I})$ are related to the field strengths $E_{i}$ by

$$
I_{i}=\frac{n_{i} \epsilon_{0} c_{0}}{2}\left|E_{i}\right|^{2}
$$

The wavevector mismatch $\Delta k_{z}$ reduces the parametric amplification. If the parametric susceptibility $\chi_{\mathrm{P}}^{(3)}$ is real (nonresonant interaction), then parametric amplification is only possible for $\kappa_{\mathrm{S}}^{*} \kappa_{\mathrm{I}}>\Delta k_{z}$. Otherwise the hyperbolic functions cosh and sinh change to the trigonometric functions cos and sin.

In the case of collinear phase-matched interaction the signal and idler intensities reduce to

$$
\begin{array}{r}
I_{\mathrm{S}}(z)=I_{\mathrm{S}}(0)\left|\cosh \left(\beta_{0} z\right)\right|^{2} \\
I_{\mathrm{I}}(z)=\frac{\omega_{\mathrm{I}} n_{\mathrm{S}}}{\omega_{\mathrm{S}} n_{\mathrm{I}}} I_{\mathrm{S}}(0)\left|\sinh \left(\beta_{0} z\right)\right|^{2}
\end{array}
$$

with

$$
\begin{aligned}
\beta_{0} & =\left(\kappa_{\mathrm{S}}^{*} \kappa_{\mathrm{I}}\right)^{1 / 2}=\left(\frac{\omega_{\mathrm{S}} \omega_{\mathrm{I}}}{n_{\mathrm{S}} n_{\mathrm{I}}}\right)^{1 / 2} \frac{D\left(\omega_{1}, \omega_{2}\right)}{2 c_{0}}\left|E_{1} E_{2}\right| \chi_{\mathrm{P}}^{(3)^{*}} \\
& =\left(\frac{\omega_{\mathrm{S}} \omega_{\mathrm{I}}}{n_{1} n_{2} n_{\mathrm{S}} n_{\mathrm{I}}} I_{1} I_{2}\right)^{1 / 2} \frac{D\left(\omega_{1}, \omega_{2}\right)}{\epsilon_{0} c_{0}^{2}} \chi_{\mathrm{P}}^{(3)^{*}}
\end{aligned}
$$

The refractive index dispersion $n(\lambda)$ requires noncollinear interaction of the light fields to achieve wavevector matching $\Delta \boldsymbol{k}=\boldsymbol{k}_{\mathrm{S}}+\boldsymbol{k}_{\mathrm{I}}-\boldsymbol{k}_{1}-\boldsymbol{k}_{2}=0$. Noncollinear beam arrangements and phase-matching wavevector diagrams for normal dispersive isotropic media are shown in Figs 1 and 2, respectively. In the case (a) of $\omega_{\mathrm{S}}>\omega_{2} \geq \omega_{1}>\omega_{\mathrm{I}}$ or $\omega_{\mathrm{I}}>\omega_{2} \geq \omega_{1}>\omega_{\mathrm{S}}$ the beam arrangement of Fig. 1a and the wavevector diagram of 

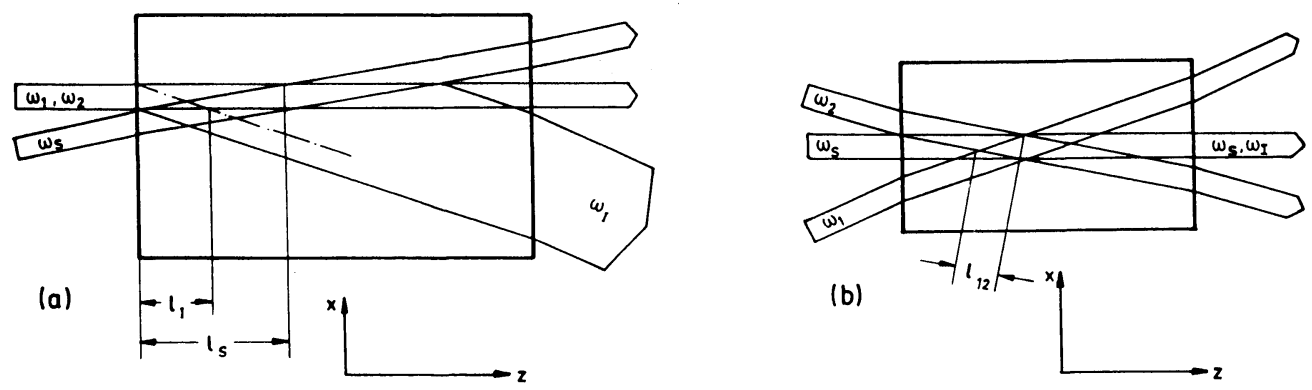

Figure 1 Schematic noncollinear phase-matched beam overlap for interaction $\omega_{1}+\omega_{2} \rightarrow \omega_{S}+\omega_{1}$ in normal dispersive media. (a) Collinear pump pulse propagation for $\omega_{1}<\omega_{1} \leq \omega_{2}<\omega_{\mathrm{S}}$ or $\omega_{\mathrm{S}}<\omega_{1} \leq \omega_{2}<\omega_{1}$. (b) Noncollinear pump pulse propagation for $\omega_{1}<\omega_{\mathrm{S}}<\omega_{2}$.

Fig. 2a apply where the pump pulses are collinear and the phase-matching angles $\varphi$ and $\psi$ are given by

$$
\varphi=\arccos \left[\frac{\left(k_{1}+k_{2}\right)^{2}+k_{\mathrm{S}}^{2}-k_{\mathrm{I}}^{2}}{2\left(k_{1}+k_{2}\right) k_{\mathrm{S}}}\right]
$$

(a)

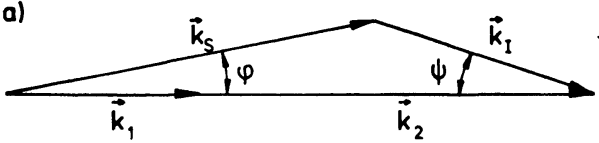

(b)

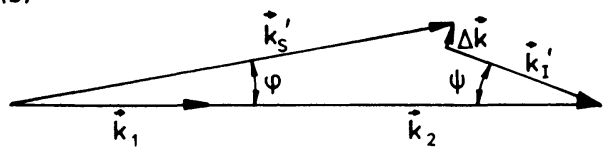

(c)

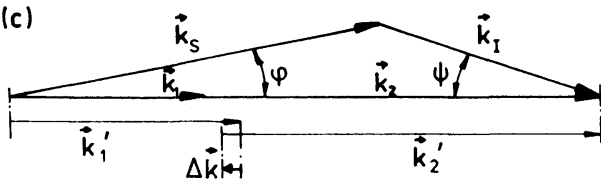

(d)

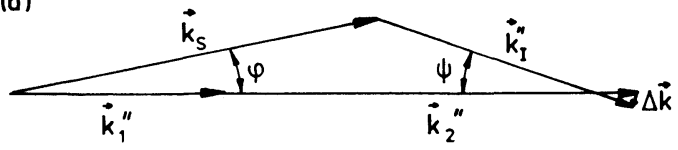

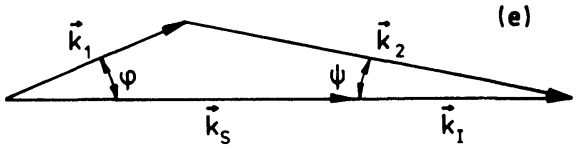

(f)

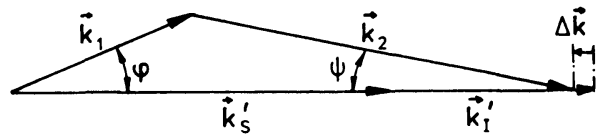

(g)

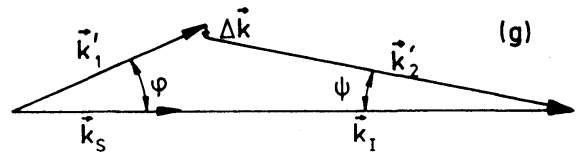

(h)

Figure 2 Wavevector diagrams. (a-d) Collinear pump pulse propagation according to Fig. 1a. (e-h) Noncollinear pump pulse propagation according to Fig. 1b. (a) and (e) Noncollinear phase-matching. (b) and (f) Phase mismatch due to frequency detuning of signal light. ' $c$ ) and (g) Phase mismatch due to frequency detuning of pump light. (d) and (h) Phase mismatch due to self-phase modulation. 
and

$$
\psi=\arccos \left[\frac{\left(k_{1}+k_{2}\right)^{2}+k_{\mathrm{I}}^{2}-k_{\mathrm{S}}^{2}}{2\left(k_{1}+k_{2}\right) k_{\mathrm{I}}}\right]
$$

In the case (b) of $\omega_{1}<\omega_{\mathrm{I}} \leq \omega_{\mathrm{S}}<\omega_{2}$ or $\omega_{1}<\omega_{\mathrm{S}} \leq \omega_{\mathrm{I}}<\omega_{2}$ the beam arrangement of Fig. $1 \mathrm{~b}$ and the wavevector diagram of Fig. 2e apply where the signal and idler light are collinear and the phase-matching angles $\varphi$ and $\psi$ are given by

$$
\varphi=\arccos \left[\frac{\left(k_{\mathrm{S}}+k_{\mathrm{I}}\right)^{2}+k_{1}^{2}-k_{2}^{2}}{2\left(k_{\mathrm{S}}+k_{\mathrm{I}}\right) k_{1}}\right]
$$

and

$$
\psi=\arccos \left[\frac{\left(k_{\mathrm{S}}+k_{\mathrm{I}}\right)^{2}+k_{2}^{2}-k_{1}^{2}}{2\left(k_{\mathrm{S}}+k_{\mathrm{I}}\right) k_{2}}\right]
$$

The noncollinear pulse propagation limits the spatial overlap of the pulses of finite beam diameter in long samples, as illustrated in Figs. 1a and 1b. We consider the situation of equal beam diameters $d$ (FWHM) of the pump pulses and the signal pulse. In case (a) (collinear pump pulses, Fig. 1a) effective spatial signal and idler overlap lengths $l_{\mathrm{S}}$ and $l_{\text {I }}$ may be defined by

$$
l_{\mathrm{S}}=d / \tan (\varphi)
$$

and

$$
l_{\mathrm{I}}=d / \tan (\psi)
$$

where $\varphi$ and $\psi$ are given by Equations $12 \mathrm{a}$ and $12 \mathrm{~b}$, respectively.

In case (b) (noncollinear pump pulses, Fig. 1b), the effective spatial overlap length may be defined by

$$
l_{12}=d / \tan (\varphi+\psi)
$$

where $\varphi$ and $\psi$ are given by Equations $13 \mathrm{a}$ and $13 \mathrm{~b}$, respectively.

For noncollinear phase-matched parametric four-photon amplification in nonabsorbing samples of length $l>l_{\mathrm{S}}, l_{\mathrm{I}}, l_{12}$ the amplification is approximately given by

$$
\frac{I_{\mathrm{S}}(l)}{I_{\mathrm{S}}(0)} \approx\left|\cosh \left(\beta_{0} l_{\mathrm{S}}\right)\right|^{2}
$$

for $\omega_{\mathrm{S}}<\omega_{1} \leq \omega_{2}\left(l_{\mathrm{S}}<l_{\mathrm{I}}\right)$

$$
\frac{I_{\mathrm{S}}(l)}{I_{\mathrm{S}}(0)} \approx\left|\cosh \left(\beta_{0} l_{\mathrm{I}}\right)\right|^{2 l_{\mathrm{S}} / l_{\mathrm{I}}} \approx \begin{cases}\left|\cosh \left[\beta_{0}\left(l_{\mathrm{I}} l_{\mathrm{S}}\right)^{1 / 2}\right]\right|^{2} & \text { if } \beta_{0} l_{\mathrm{I}} \ll 1 \\ \left|\cosh \left(\beta_{0} l_{\mathrm{S}}\right)\right|^{2} & \text { if } \beta_{0} l_{\mathrm{I}} \gg 1\end{cases}
$$

for $\omega_{\mathrm{S}}>\omega_{2} \geq \omega_{1}\left(l_{\mathrm{S}}>l_{\mathrm{I}}\right) ;$ and

$$
\frac{I_{\mathrm{S}}(l)}{I_{\mathrm{S}}(0)} \approx\left|\cosh \left(\beta_{0} l_{12}\right)\right|^{2}
$$

for $\omega_{1}<\omega_{\mathrm{S}}<\omega_{2}\left(l_{12}<l_{\mathrm{I}}, l_{\mathrm{S}}\right)$. 
Equation 17 takes into account that parametric four-photon amplification continues in the region $l_{\mathrm{I}}<l<l_{\mathrm{S}}$ with build-up of idler light from quantum noise [15], i.e. at $z=l_{\mathrm{I}}$ the signal is $I_{\mathrm{S}}\left(z=l_{\mathrm{I}}\right) \approx I_{\mathrm{S}}(0)\left|\cosh \left(\beta_{0} l_{\mathrm{I}}\right)\right|^{2} ;$ at $z=2 l_{\mathrm{I}}$ it is $I_{\mathrm{S}}\left(z=2 l_{\mathrm{I}}\right) \approx$ $I_{\mathrm{S}}\left(z=l_{\mathrm{S}}\right)\left|\cosh \left(\beta_{0} l_{\mathrm{I}}\right)\right|^{2} \approx I_{\mathrm{S}}(0)\left|\cosh \left(\beta_{0} l_{\mathrm{I}}\right)\right|^{4}$; and at $z=n l_{\mathrm{I}}=\left(l_{\mathrm{S}} / l_{\mathrm{I}}\right) l_{\mathrm{I}}=l_{\mathrm{S}}$ it is $I_{\mathrm{S}}\left(n l_{\mathrm{I}}\right)=$ $I_{\mathrm{S}}\left[(n-1) l_{\mathrm{I}}\right]\left|\cosh \left(\beta_{0} l_{\mathrm{I}}\right)\right|^{2} \approx I_{\mathrm{S}}(0)\left|\cosh \left(\beta_{0} l_{\mathrm{I}}\right)\right|^{2 n}=I_{\mathrm{S}}(0)\left|\cosh \left(\beta_{0} l_{\mathrm{I}}\right)\right|^{2 l_{\mathrm{S}} / l_{\mathrm{I}}}$. Approximation (17a) is obtained by Taylor expansion of cosh, while approximation $(17 \mathrm{~b})$ is obtained by approximating cosh with exp.

In media absorbing at the signal or idler wavelength the effective interaction length $l_{\alpha}$ is limited to

$$
l_{\alpha} \approx \begin{cases}\min \left[\alpha_{\mathrm{S}}^{-1},\left(\alpha_{\mathrm{S}} \alpha_{\mathrm{I}}\right)^{-1 / 2}\right] & \text { if } \beta_{0} \alpha_{\mathrm{I}}^{-1} \ll 1 \\ \alpha_{\mathrm{S}}^{-1} & \text { if } \beta_{0} \alpha_{\mathrm{I}}^{-1} \gg 1\end{cases}
$$

and the sample length $l$ should be chosen to be $l \leq l_{\alpha}$. Relation (19) was obtained by considering the structure of Equation 17.

The pump pulses and the signal and idler pulses move with slightly different group velocities $v_{\mathrm{g}, i}=c_{0} / n_{\mathrm{g}, i}(i=1,2, \mathrm{~S}, \mathrm{I})$ where the group refractive index $n_{\mathrm{g}, i}$ is given by [10]

$$
n_{\mathrm{g}, i}=n+\omega_{i} \frac{\partial n}{\partial \omega_{i}}=n-\lambda_{i} \frac{\partial n}{\partial \lambda_{i}}
$$

After a propagation distance $l$, the temporal delay $\delta t_{1, \mathrm{~S}}$ between pump pulse 1 and signal pulse $\mathrm{S}$ is

$$
\delta t_{1, \mathrm{~S}}=\left|\frac{l}{v_{\mathrm{g}, 1}}-\frac{l}{\cos (\varphi) v_{\mathrm{g}, \mathrm{S}}}\right|=\frac{l}{c_{0}}\left|n_{\mathrm{g}, 1}-\frac{n_{\mathrm{g}, \mathrm{S}}}{\cos (\varphi)}\right|
$$

for collinear pump pulse propagation (case (a), Fig. 1a), and

$$
\delta t_{1, \mathrm{~S}}=\left|\frac{l}{\cos (\varphi) v_{\mathrm{g}, 1}}-\frac{l}{v_{\mathrm{g}, \mathrm{S}}}\right|=\frac{l}{c_{0}}\left|\frac{n_{\mathrm{g}, 1}}{\cos (\varphi)}-n_{\mathrm{g}, \mathrm{S}}\right|
$$

for noncollinear pump pulse propagation (case (b), Fig. 1b).

The temporal spread between the pump pulses $\delta t_{1,2}$ is

$$
\delta t_{1,2}=\frac{l}{c_{0}}\left|n_{\mathrm{g}, 1}-n_{\mathrm{g}, 2}\right|
$$

in case (a) (collinear pump pulse propagation), and

$$
\delta t_{1,2}=\frac{l}{c_{0}}\left|\frac{n_{\mathrm{g}, 1}}{\cos (\varphi)}-\frac{n_{\mathrm{g}, 2}}{\cos (\psi)}\right|
$$

in case (b) (noncollinear pump pulse propagation).

As long as $\delta t_{1, \mathrm{~S}}$ or $\delta t_{1,2}$ are small compared to the pulse duration $\Delta t$ of the pump and signal pulses (same duration $\Delta t$ is assumed for pump and signal pulses) the effect of the different group velocities of the interacting pulses may be neglected. Otherwise the time spreading limits the effective sample length $l_{t}$ to

$$
l_{t} \approx \min \left(\frac{\Delta t}{\left|\delta t_{1, \mathrm{~S}} / l\right|}, \frac{\Delta t}{\left|\delta t_{1,2} / l\right|}\right)
$$


The noncollinear pulse propagation, the optical absorption, and the temporal spread limit the parametric four-photon amplification to an effective length

$$
l_{\mathrm{eff}}=\min \left[\left(l_{\mathrm{S}} l_{\mathrm{I}}\right)^{1 / 2} \text { or } l_{12}, l_{\alpha}, l_{t}, l\right]
$$

(see Equations 14, 15, 17a, 19, 22).

Exact noncollinear phase-matching is only possible for monochromatic waves. The dependence of the wavevector mismatch on the spectral bandwidths of the pump pulses and the signal pulse determines the bandwidth limitations of the parametric amplification for short-duration and broadband pulses.

The phase mismatch $\Delta \phi=\Delta k_{z} z$ of Equations 1 to 4 changes to $\Delta \phi=\Delta \boldsymbol{k r}=$ $\Delta k_{z} z+\Delta k_{x} x$ in the case of noncollinear propagation where $x$ is the propagation coordinate transverse to the main propagation coordinate $z$. The parametric gain factor $\beta$ (Equation 5) remains unchanged. Parametric amplification is only possible when $\beta_{0}>\Delta k_{z}$. The total transverse phase mismatch should be $\Delta k_{x} d_{\mathrm{eff}}=\Delta k_{x} \tan (\varphi) l_{\mathrm{eff}}<\pi$ to avoid reduction of parametric amplification $[15,37]$. For $\Delta k_{z}=0$ one speaks of longitudinally phase-matched interaction or Čerenkov-like interaction [15, 38-40], while in the case of $\Delta k_{x}=0$ one speaks of transversely phase-matched interaction. Transverse wavevector mismatch $\Delta k_{x} \neq 0$ practically does not influence the parametric four-photon interaction as long as the transverse phase mismatch is $\Delta \phi_{x}=\Delta k_{x} d_{\text {eff }} \ll \pi$.

Wavevector diagrams of noncollinear interaction with residual wavevector mismatch $\Delta \boldsymbol{k}$ are illustrated in Figs. $2 \mathrm{~b}$ to $\mathrm{d}$ and $\mathrm{f}$ to $\mathrm{h}$. The angles $\varphi$ and $\psi$ are kept the same as in the noncollinear phase-matched situations of Fig. 2a (corresponding to Fig. 1a) and Fig. 2e (corresponding to Fig. 1b). In all cases the idler frequency is adjusted to satisfy energy conservation. A detuning of the signal frequency from $\omega_{\mathrm{S}}$ to $\omega_{\mathrm{S}}^{\prime}=\omega_{\mathrm{S}}+\Delta \omega_{\mathrm{S}} / 2$ gives a wavevector mismatch of

$$
\begin{aligned}
& \Delta k_{z}=k_{\mathrm{S}}^{\prime} \cos \varphi+k_{\mathrm{I}}^{\prime} \cos \psi-k_{1}-k_{2}=\kappa_{\mathrm{SI}, z} \Delta \tilde{\nu}_{\mathrm{S}} \\
& \Delta k_{x}=k_{\mathrm{S}}^{\prime} \sin \varphi-k_{\mathrm{I}}^{\prime} \sin \psi=\kappa_{\mathrm{SI}, x} \Delta \tilde{\nu}_{\mathrm{S}}
\end{aligned}
$$

in the case of collinear pump pulse propagation (Fig. 2b), and of

$$
\begin{aligned}
\Delta k_{z} & =k_{\mathrm{S}}^{\prime}+k_{\mathrm{I}}^{\prime}-k_{\mathrm{S}}-k_{\mathrm{I}} \\
& =k_{\mathrm{S}}^{\prime}+k_{\mathrm{I}}^{\prime}-k_{1} \cos \varphi-k_{2} \cos \psi=\kappa_{\mathrm{SI}, z} \Delta \tilde{\nu}_{\mathrm{S}} \\
\Delta k_{x} & =0
\end{aligned}
$$

in the case of noncollinear pump pulse propagation (Fig. 2f). In Equations 24 $k_{\mathrm{S}}^{\prime}=n\left(\omega_{\mathrm{S}}^{\prime}\right) \omega_{\mathrm{S}}^{\prime} / c_{0}$ and $k_{\mathrm{I}}^{\prime}=n\left(\omega_{\mathrm{I}}^{\prime}\right) \omega_{\mathrm{I}}^{\prime} / c_{0}$ with $\omega_{\mathrm{I}}^{\prime}=\omega_{\mathrm{I}}-\Delta \omega_{\mathrm{S}} / 2 . \kappa_{\mathrm{SI}}=\Delta k_{z} / \Delta \tilde{\nu}_{\mathrm{S}}$ is the wavevector mismatch coefficient.

Bandwidth-limited pump pulses of a finite spectral width of $\Delta \omega=\Delta \omega_{1}=\Delta \omega_{2}$ cause an effective wavevector mismatch of

$$
\begin{aligned}
& \Delta k_{z}=k_{1}^{\prime}+k_{2}^{\prime}-k_{1}-k_{2}=\kappa_{12, z} \Delta \tilde{\nu} \\
& \Delta k_{x}=0
\end{aligned}
$$


in the case collinear pump pulse propagation (Fig. 2c), and of

$$
\begin{aligned}
& \Delta k_{z}=k_{\mathrm{S}}+k_{\mathrm{I}}-k_{1}^{\prime} \cos \varphi-k_{2}^{\prime} \cos \psi=\kappa_{12, z} \Delta \tilde{\nu} \\
& \Delta k_{x}=k_{2}^{\prime} \sin \psi-k_{1}^{\prime} \sin \varphi=\kappa_{12, x} \Delta \tilde{\nu}
\end{aligned}
$$

in the case of noncollinear pump pulse propagation (Fig. 2g). In Equations 25 $k_{1}^{\prime}=n\left(\omega_{1}^{\prime}\right) \omega_{1}^{\prime} / c_{0}$ and $k_{2}^{\prime}=n\left(\omega_{2}^{\prime}\right) \omega_{2}^{\prime} / c_{0}$ with $\omega_{1}^{\prime}=\omega_{1}+\Delta \omega / 2$ and $\omega_{2}^{\prime}=\omega_{2}-\Delta \omega / 2$. In the degenerate case, $\omega_{1}=\omega_{2}$, the wavevector mismatch caused by the finite spectral bandwidth of the pump pulse is negligible (Equation 25a).

For self-phase-modulated pump pulses (see below) the carrier frequency chirps with time [1]. A spectral broadening of $\Delta \nu_{\mathrm{SPM}}$ causes an effective wavevector mismatch of

$$
\begin{aligned}
& \Delta k_{z}=k_{\mathrm{S}} \cos \varphi+k_{\mathrm{I}}^{\prime \prime} \cos \psi-k_{1}^{\prime \prime}-k_{2}^{\prime \prime}=\kappa_{\mathrm{SPM}, z} \Delta \tilde{\nu}_{\mathrm{SPM}} \\
& \Delta k_{x}=k_{\mathrm{S}} \sin \varphi-k_{\mathrm{I}}^{\prime \prime} \sin \psi=\kappa_{\mathrm{SPM}, x} \Delta \tilde{\nu}_{\mathrm{SPM}}
\end{aligned}
$$

in the case of collinear pump pulse propagation (Fig. 2d), and of

$$
\begin{aligned}
& \Delta k_{z}=k_{\mathrm{S}}+k_{\mathrm{I}}^{\prime \prime}-k_{1}^{\prime \prime} \cos \varphi-k_{2}^{\prime \prime} \cos \psi=\kappa_{\mathrm{SPM}, z} \Delta \tilde{\nu}_{\mathrm{SPM}} \\
& \Delta k_{x}=k_{2}^{\prime \prime} \sin \psi-k_{1}^{\prime \prime} \sin \varphi=\kappa_{\mathrm{SPM}, x} \Delta \tilde{\nu}_{\mathrm{SPM}}
\end{aligned}
$$

in the case of noncollinear pump pulse propagation (Fig. 2h). In Equations 26 $k_{1}^{\prime \prime}=n_{1}\left(\omega_{1}^{\prime \prime}\right) \omega_{1}^{\prime \prime} / c_{0}, \quad k_{2}^{\prime \prime}=n_{2}\left(\omega_{2}^{\prime \prime}\right) \omega_{2}^{\prime \prime} / c_{0}, \quad$ and $\quad k_{\mathrm{I}}^{\prime \prime}=n\left(\omega_{\mathrm{I}}^{\prime \prime}\right) \omega_{\mathrm{I}}^{\prime \prime} / c_{0} \quad$ with $\quad \omega_{1}^{\prime \prime}=\omega_{1}+\Delta \omega_{\mathrm{SPM}} / 2$, $\omega_{2}^{\prime \prime}=\omega_{2}+\Delta \omega_{\mathrm{SPM}} / 2$, and $\omega_{\mathrm{I}}^{\prime \prime}=\omega_{\mathrm{I}}-\Delta \omega_{\mathrm{SPM}}=\omega_{1}^{\prime \prime}+\omega_{2}^{\prime \prime}-\omega_{\mathrm{S}}$.

The parametric amplification of broadband signal light is limited to

$$
\Delta \tilde{\nu}_{\mathrm{S}} \leq \frac{\beta_{0}}{\left|\kappa_{\mathrm{SI}, z}\right|}
$$

according to Equations 5, 11, 24a and 24c.

The spectral width $\Delta \tilde{\nu}\left(\Delta \tilde{\nu}_{1}=\Delta \tilde{\nu}_{2}\right)$ of the pump pulses should be so that $\Delta k_{z}<\beta_{0}$ (Equations 5, 11 and 25a, 25c) in order to achieve parametric amplification over the whole spectral pump pulse width. This condition limits the pump pulse spectral width $\Delta \tilde{\nu}$ for whole-bandwidth parametric amplification to

$$
\Delta \tilde{\nu} \leq \frac{\beta_{0}}{\left|\kappa_{12, z}\right|}
$$

For self-phase-modulated pulses of chirp width $\Delta \tilde{\nu}_{\mathrm{SPM}}$, parametric amplification over the full chirped range occurs only if

$$
\Delta \tilde{\nu}_{\mathrm{SPM}} \leq \frac{\beta_{0}}{\left|\kappa_{\mathrm{SPM}, z}\right|}
$$

\section{Numerical simulations}

The efficiency of noncollinear phase-matched parametric four-photon amplification in the isotropic media heavy water, fused silica, and Schott glass SF10 is analysed. Calculations are carried out for pump pulses at wavelengths of $\lambda_{\mathrm{L}}=1054 \mathrm{~nm}$ and $\lambda_{\mathrm{SH}}=527 \mathrm{~nm}$, corresponding to the fundamental and second harmonic wavelengths of an $\mathrm{Nd}$ : glass laser. The results apply as well to the wavelengths $1064 \mathrm{~nm}$ and $532 \mathrm{~nm}$ of $\mathrm{Nd}$ : YAG lasers. 


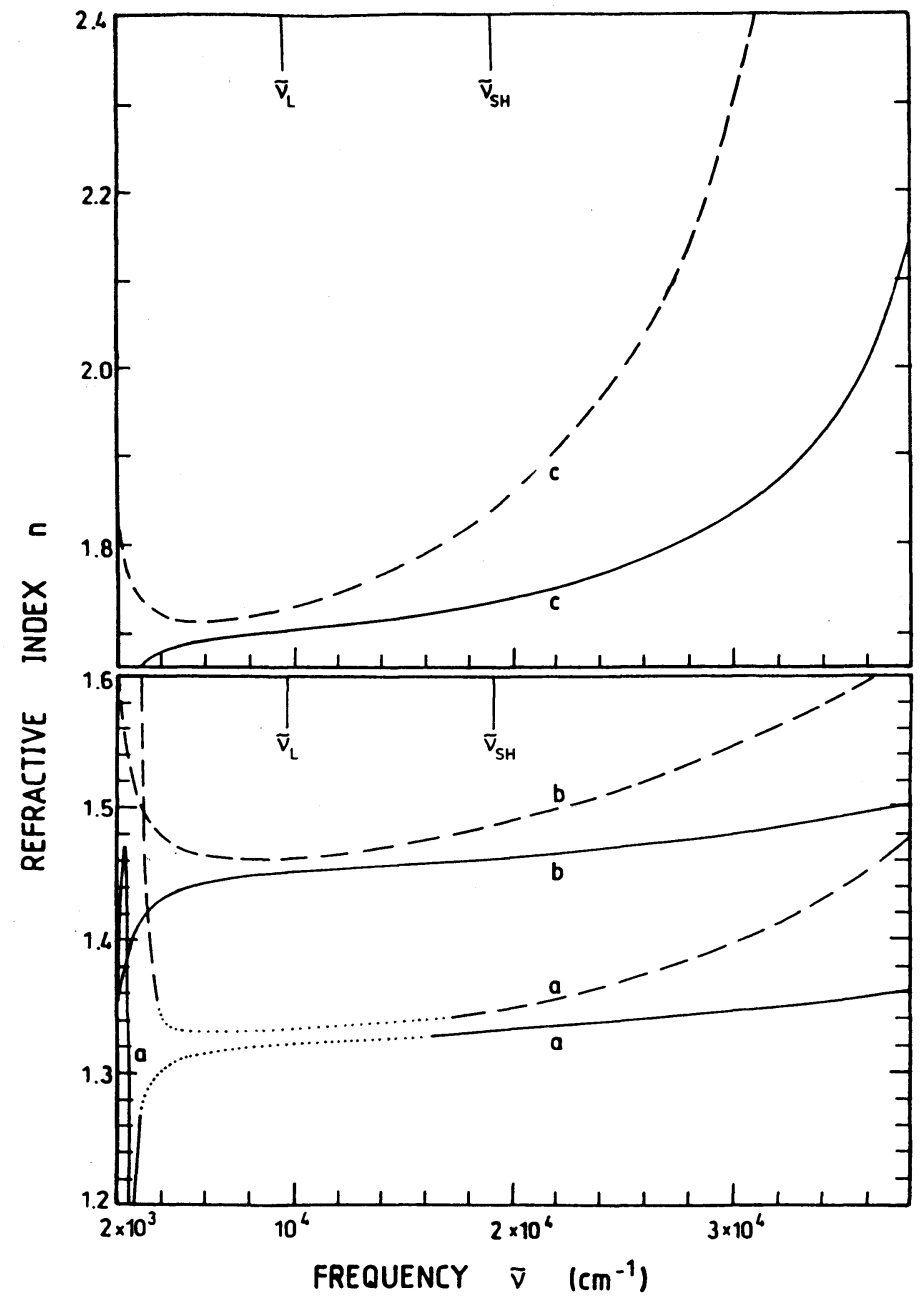

Figure 3 Dispersion of phase refractive indices $n$ (solid curves) and group refractive indices $n_{\mathrm{g}}$ (dashed curves). Curves a: $\mathrm{D}_{2} \mathrm{O}$ (data from $[41,42]$ ). Dotted parts are extrapolated. $n_{\mathrm{g}}(\tilde{\nu})$ below $3000 \mathrm{~cm}^{-1}$ is not included. Curves b: fused silica (data from [60]). Curves c: SF10 (data from [66]).

The interaction processes (I) $\omega_{\mathrm{L}}+\omega_{\mathrm{L}} \rightarrow \omega_{\mathrm{S}}+\omega_{\mathrm{I}}$, (II) $\omega_{\mathrm{SH}}+\omega_{\mathrm{SH}} \rightarrow \omega_{\mathrm{S}}+\omega_{\mathrm{I}}$, and (III) $\omega_{\mathrm{L}}+\omega_{\mathrm{SH}} \rightarrow \omega_{\mathrm{S}}+\omega_{\mathrm{I}}$ are considered.

The wavelength dependence of the refractive indices $n(\lambda)$ of the media investigated is displayed in the solid curves of Fig. 3. The refractive index dispersion of the glasses is approximated by

$$
n^{2}(\lambda)-1=\sum_{i=1}^{3} \frac{B_{i} \lambda^{2}}{\lambda^{2}-C_{i}}
$$

The fit parameters $B_{i}$ and $C_{i}$ are listed in Table $\mathrm{I}$. The refractive indices of $\mathrm{D}_{2} \mathrm{O}$ are taken from [41] (visible and UV range) and from [42] (infrared region). The refractive index 
TABLE I Parameters of refractive index dispersion formula (Equation 28) for glasses

\begin{tabular}{lll}
\hline Material & $\mathrm{SiO}_{2}$ & $\mathrm{SF} 10$ \\
\hline$B_{1}$ & 0.6961663 & 1.61625977 \\
$B_{2}$ & 0.4079426 & 0.259229334 \\
$B_{3}$ & 0.8974794 & 1.07762317 \\
$C_{1}$ & $4.679148 \times 10^{-3}$ & $1.27534559 \times 10^{-2}$ \\
$C_{2}$ & $1.351206 \times 10^{-2}$ & $5.81983954 \times 10^{-2}$ \\
$C_{3}$ & 97.93400 & 116.607680 \\
Reference & {$[65]$} & {$[66]$} \\
\hline
\end{tabular}

dispersion of the media increases in the order $\mathrm{D}_{2} \mathrm{O}, \mathrm{SiO}_{2}, \mathrm{SF} 10$. The dashed curves in Fig. 3 show the wavelength dependence of the group refractive indices $n_{\mathrm{g}}(\lambda) . n_{\mathrm{g}}(\lambda)$ is calculated from $n(\lambda)$ by use of Equation 20. Below $3000 \mathrm{~cm}^{-1}$ the group refractive index dispersion of $\mathrm{D}_{2} \mathrm{O}$ is not included.

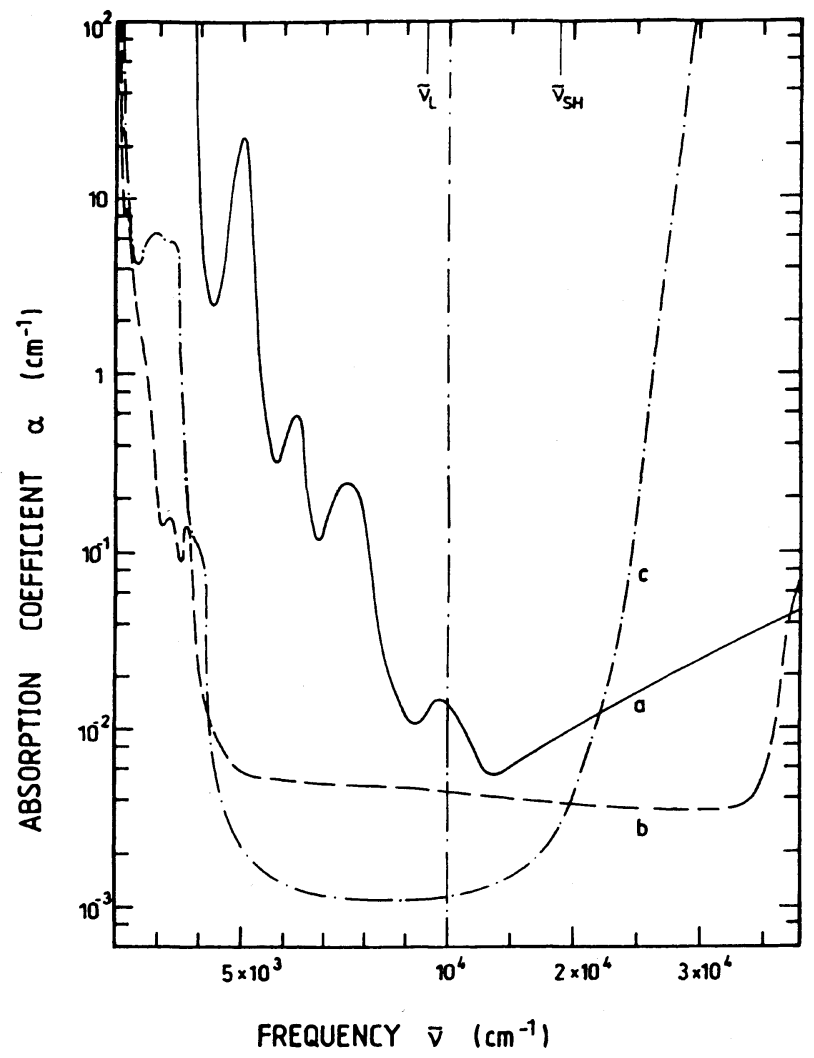

Figure 4 Wavelength dependence of absorption coefficients $\alpha$. Curve a: $\mathrm{D}_{2} \mathrm{O}$ (own measurements). Curve b: $\mathrm{SiO}_{2}$ grade Infrasil (from [43]). Curve c: Schott glass SF10 (from [66] and own measurements for $\tilde{\nu}<4300 \mathrm{~cm}^{-1}$ and $\tilde{\nu}>27400 \mathrm{~cm}^{-1}$ ). 
The absorption coefficient spectra $\alpha(\lambda)$ of the investigated media are shown in Fig. 4. The displayed absorption spectrum of fused silica belongs to the grade Infrasil having weak infrared absorption [43]. Of the investigated substances, $\mathrm{D}_{2} \mathrm{O}$ has the strongest absorption in the near infrared, while the flint glass Schott SF10 already begins to absorb strongly in the violet spectral region.

The noncollinear phase-matching angles $\varphi$ belonging to the three interaction processes I, II and III versus frequency are displayed in Fig. 5 (appropriate Equations 12a and 13a; collinear pump pulse propagation with the exception of interaction III in the region $\left.\omega_{\mathrm{L}}<\omega_{\mathrm{S}}<\omega_{\mathrm{SH}}\right)$. The phase-matching angles increase in the order $\mathrm{D}_{2} \mathrm{O}, \mathrm{SiO}_{2}$ and $\mathrm{SF} 10$.

The normalized spatial overlap lengths $l_{\text {ov }} / d$ versus frequency are displayed in Fig. 6 for the various interaction processes $\left(l_{\mathrm{ov}}=l_{\mathrm{S}}\right.$, Equation $14 \mathrm{a}$, for processes I and II, and for $\omega_{\mathrm{S}}<\omega_{\mathrm{L}}$ and $\omega_{\mathrm{S}}>\omega_{\mathrm{SH}}$ in the case of process III; $l_{\mathrm{ov}}=l_{12}$, Equation 15, in the case of process III in the region $\left.\omega_{\mathrm{L}}<\omega_{\mathrm{S}}<\omega_{\mathrm{SH}}\right)$. Around the pump laser frequencies the spatial overlap lengths are large. The vertical lines indicate the borders outside which no noncollinear phase-matching is possible for collinear pump pulse propagation (see Fig. 5).

The normalized temporal spreading $\delta t_{1, \mathrm{~S}} / l$ between signal pulse and pump pulse of frequency $\omega_{1}\left(\omega_{1} \leq \omega_{2}\right.$, Equation 21a) is plotted in Fig. 7. Apart from the position $\omega_{\mathrm{S}}=\omega_{1}$, there is no time spreading at wavelengths of group velocity matching, i.e.

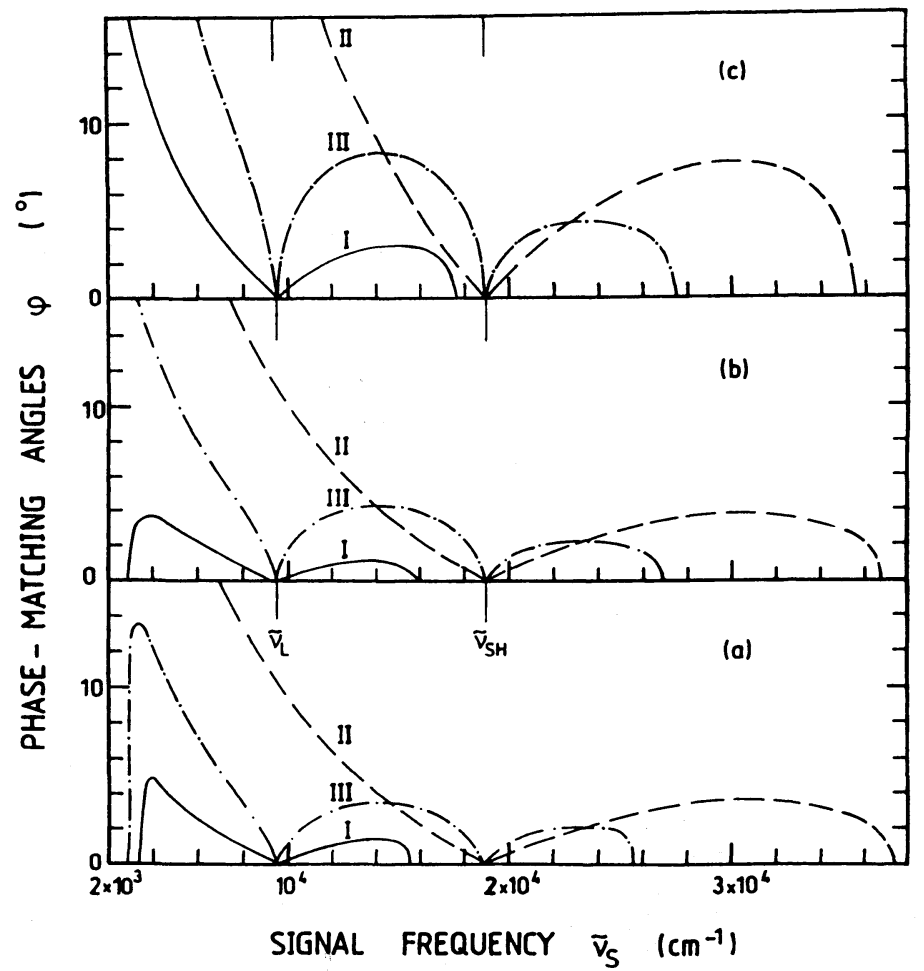

Figure 5 Phase-matching angles $\varphi$ between $\boldsymbol{k}_{1}$ and $\boldsymbol{k}_{\mathrm{S}}$ for the processes (I) $\omega_{\mathrm{L}}+\omega_{\mathrm{L}} \rightarrow \omega_{\mathrm{S}}+\omega_{\mathrm{I}}$ (solid curves), (II) $\omega_{\mathrm{SH}}+\omega_{\mathrm{SH}} \rightarrow \omega_{\mathrm{S}}+\omega_{\mathrm{I}}$ (dashed curves), and (III) $\omega_{\mathrm{L}}+\omega_{\mathrm{SH}} \rightarrow \omega_{\mathrm{S}}+\omega_{\mathrm{I}}$ (dash-dotted curves). (a) $\mathrm{D}{ }_{2} \mathrm{O}$; 


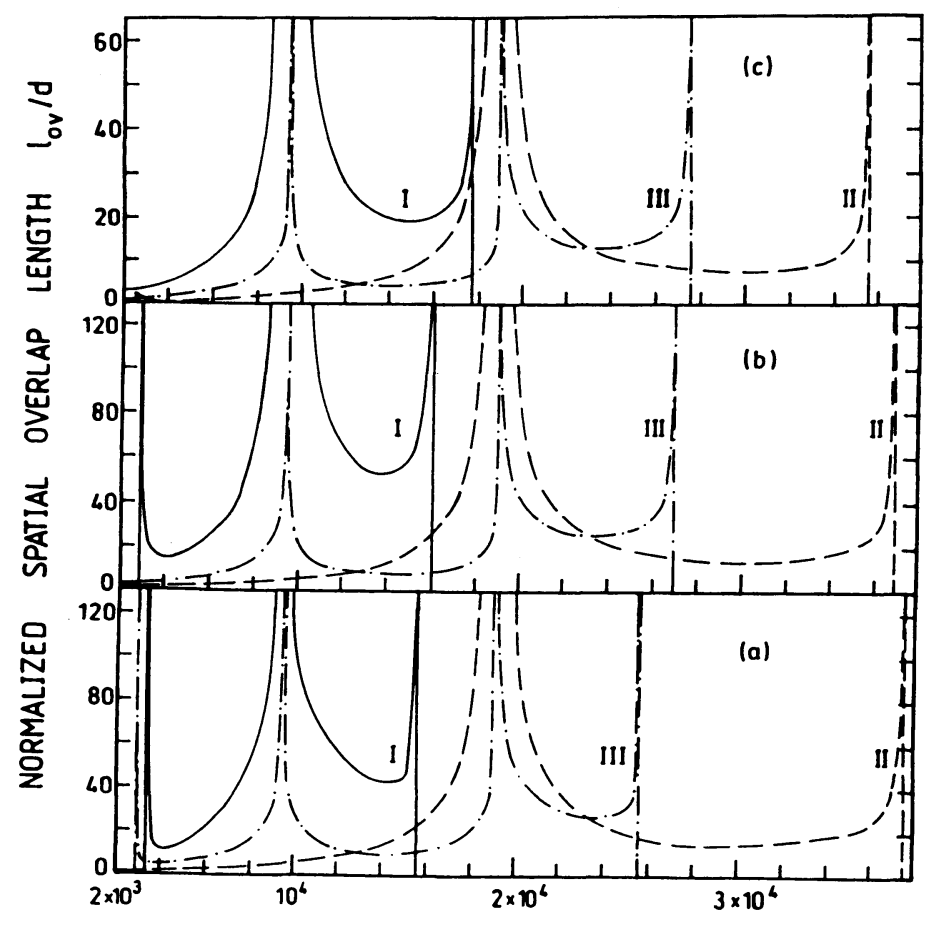

SIGNAL FREQUENCY $\bar{\nabla}_{S}\left(\mathrm{~cm}^{-1}\right)$

Figure 6 Normalized spatial overlap lengths $I_{\text {ov }} / d$ for the interaction processes $(I) \omega_{\mathrm{L}}+\omega_{\mathrm{L}} \rightarrow \omega_{\mathrm{S}}+\omega_{\mathrm{I}}$ (solid curves), (II) $\omega_{\mathrm{SH}}+\omega_{\mathrm{SH}} \rightarrow \omega_{\mathrm{S}}+\omega_{\mathrm{I}}$ (dashed curves), and (III) $\omega_{\mathrm{L}}+\omega_{\mathrm{SH}} \rightarrow \omega_{\mathrm{S}}+\omega_{\mathrm{I}}$ (dash-dotted curves). (a) $\mathrm{D}_{2} \mathrm{O}$; (b) fused silica; (c) SF10 glass. $\lambda_{\mathrm{L}}=1054 \mathrm{~nm}$. $\lambda_{\mathrm{SH}}=527 \mathrm{~nm}$.

$n_{\mathrm{g}, 1}=n_{\mathrm{g}, \mathrm{S}} / \cos (\varphi)$ (Equation 21a) or $n_{\mathrm{g}, \mathrm{S}}=n_{\mathrm{g}, 1} / \cos (\varphi)$ (Equation 21b). The time spreading should be short compared to the signal and pump pulse duration in order to avoid a reduction of amplification efficiency. The normalized temporal spreading $\delta t_{1,2} / l$ (Equations 21c and d) for the interaction process III is included in Fig. 7 (dotted curves).

In Fig. 8 normalized parametric amplification factors $\beta_{0}^{\prime}=\beta_{0} /\left(I_{1} I_{2}\right)^{1 / 2}$ (Equation 11) are plotted versus frequency. The parametric gain factor $G_{0}=\beta_{0} l_{\text {eff }}$ is obtained from the normalized amplification factor $\beta_{0}^{\prime}$ by multiplication with the effective pump pulse intensity $\left(I_{1} I_{2}\right)^{1 / 2}$ and the effective sample length $l_{\text {eff }}$ (Equation 23).

As examples we estimate for fused silica a gain factor of $G_{0}=1$ at $\tilde{\nu}_{\mathrm{S}}=5000 \mathrm{~cm}^{-1}$ and $\tilde{\nu}_{\mathrm{S}}=14000 \mathrm{~cm}^{-1}$ applying $I_{0 \mathrm{~L}}=10^{11} \mathrm{~W} \mathrm{~cm}^{-2}$ and $l_{\text {eff }}=1 \mathrm{~cm}$ to the interaction process (I) $\left(\omega_{\mathrm{L}}+\omega_{\mathrm{L}} \rightarrow \omega_{\mathrm{S}}+\omega_{\mathrm{I}}\right)$. For the interaction process (II) $\left(\omega_{\mathrm{SH}}+\omega_{\mathrm{SH}} \rightarrow \omega_{\mathrm{S}}+\omega_{\mathrm{I}}\right)$ we calculate $G_{0}\left(\mathrm{SiO}_{2}, I_{0 \mathrm{~L}}=10^{11} \mathrm{~W} \mathrm{~cm}{ }^{-2}, l_{\text {eff }}=1 \mathrm{~cm}\right) \approx 2.2$ at $\tilde{\nu}_{\mathrm{S}}=13000 \mathrm{~cm}^{-1}$ and $\tilde{\nu}_{\mathrm{S}}=25000 \mathrm{~cm}^{-1}$. Considering the interaction process (III) $\left(\omega_{\mathrm{L}}+\omega_{\mathrm{SH}} \rightarrow \omega_{\mathrm{S}}+\omega_{\mathrm{I}}\right)$ we find $G_{0}\left(\mathrm{SiO}_{2}\right.$, $\left.I_{0 \mathrm{~L}}=I_{0, \mathrm{SH}}=10^{11} \mathrm{~W} \mathrm{~cm}^{-2}, \quad l_{\mathrm{eff}}=1 \mathrm{~cm}\right)=3$ at $\tilde{\nu}_{\mathrm{S}}=7500 \mathrm{~cm}^{-1}$ and $\tilde{\nu}_{\mathrm{S}}=21000 \mathrm{~cm}^{-1}$ $\left(G_{0}=1\right.$ corresponds to $I_{\mathrm{S}}(l) / I_{\mathrm{S}}(0)=2.38, G_{0}=3$ is equivalent to $I_{\mathrm{S}}(l) / I_{\mathrm{S}}(0)=101$, and $G_{0}=10$ gives $I_{\mathrm{S}}(l) / I_{\mathrm{S}}(0)=1.2 \times 10^{8}$ according to Equation 9).

The $\chi_{\mathrm{P}}^{(3)}$ values used in the calculation of $\beta_{0}^{\prime}$ were previously determined by thirdharmonic generation studies [44]. For $\mathrm{D}_{2} \mathrm{O}$ the same $\chi_{\mathrm{P}}^{(3)}$ value as for $\mathrm{H}_{2} \mathrm{O}$ is assumed. 


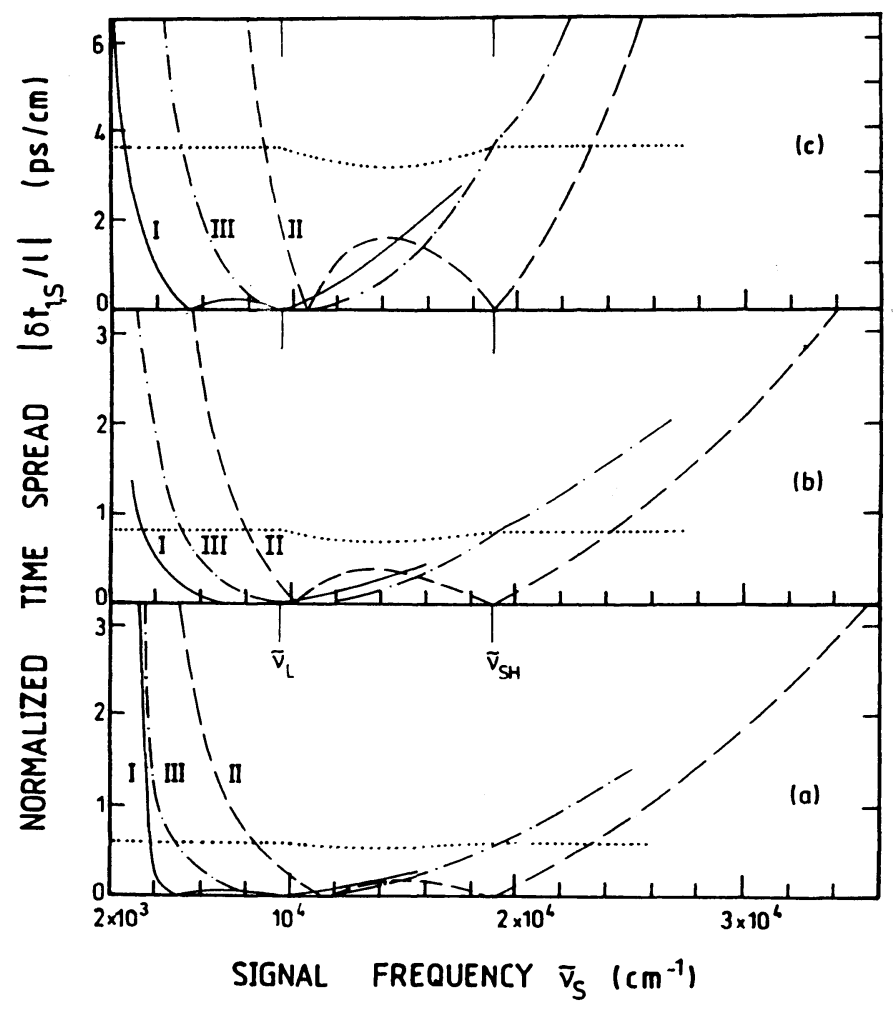

Figure 7 Normalized time spread $\left|\delta t_{1, S} / I\right|$ between pump pulse of longest wavelength and signal pulse for the interaction processes (I) $\omega_{\mathrm{L}}+\omega_{\mathrm{L}} \rightarrow \omega_{\mathrm{S}}+\omega_{\mathrm{I}}$ (solid curves), (II) $\omega_{\mathrm{SH}}+\omega_{\mathrm{SH}} \rightarrow \omega_{\mathrm{S}}+\omega_{1}$ (dashed curves), and (III) $\omega_{\mathrm{L}}+\omega_{\mathrm{SH}} \rightarrow \omega_{S}+\omega_{\mathrm{I}}\left(\omega_{1}=\omega_{\mathrm{L}}\right.$, dash-dotted curves). Dotted curves show $\left|\delta t_{1,2} / /\right|$ for interaction process (III). (a) $\mathrm{D}_{2} \mathrm{O}$; (b) fused silica; (c) SF10 glass.

The applied nonlinear susceptibilities $\chi_{\mathbf{P}}^{(3)}$ represent the nonresonant electronic contributions $\chi_{\mathrm{NR}}^{(3)}$. They increase in the order $\mathrm{D}_{2} \mathrm{O}, \mathrm{SiO}_{2}, \mathrm{SF} 10$. The frequency dependence of $\chi_{\mathrm{P}}^{(3)}=\chi_{x x x x}^{(3)}\left(-\omega_{\mathrm{S}} ; \omega_{1}, \omega_{2},-\omega_{\mathrm{I}}\right)$ is neglected (for frequency dependences see, e.g., [1, 2, 12, $35,45,46])$. In the vicinity of Raman transitions $\left|\omega_{S}-\omega_{1}\right|=\left|\omega_{2}-\omega_{I}\right|=\omega_{\mathrm{v}}$, where $\omega_{\mathrm{v}}$ is the vibrational angular frequency, the third-order nonlinear susceptibility is given by $\chi_{x x x x}^{(3)}\left(-\omega_{\mathrm{S}} ; \omega_{1}, \omega_{2},-\omega_{\mathrm{I}}\right)=\chi_{\mathrm{NR}}^{(3)}+\chi_{\mathrm{R}, x x x x}^{(3)}\left(-\omega_{\mathrm{S}} ; \omega_{1}, \omega_{2},-\omega_{\mathrm{I}}\right)$ (difference frequency resonance) $[1-3,12,15,47]$ and the parametric four-photon interaction is called coherent antiStokes-Raman scattering (CARS) or coherent Stokes-anti-Stokes coupling [1-3, 4750] (enhancement of parametric amplification around $\omega_{\mathrm{S}}=\omega_{i}-\omega_{\mathrm{v}}+\Delta \omega_{\mathrm{v}}$ and $\omega_{\mathrm{S}}=\omega_{i}+\omega_{\mathrm{v}}-\Delta \omega_{\mathrm{v}}$, reduction of parametric amplification around $\omega_{\mathrm{S}}=\omega_{i}-\omega_{\mathrm{v}}-\Delta \omega_{\mathrm{v}}$ and $\omega_{\mathrm{S}}=\omega_{i}+\omega_{\mathrm{v}}+\Delta \omega_{\mathrm{v}} ; \omega_{i}=\omega_{1}$ or $\omega_{2}, \Delta \omega_{\mathrm{v}}$ is the spectral width of Raman line).

In regions where $\omega_{\mathrm{I}}$ or $\omega_{\mathrm{S}}$ approach vibrational (in the infrared spectral region) or electronic transition frequencies (in the ultraviolet spectral region) single-frequency resonance contributions to $\chi_{x x x x}^{(3)}\left(-\omega_{\mathrm{S}} ; \omega_{1}, \omega_{2},-\omega_{\mathrm{I}}\right)$ become important $[1,2,12,35]$. In these frequency regions the linear absorption becomes important that is not included in the equation system $(1,2)$ (for inclusion of absorption see, e.g., [12]). 


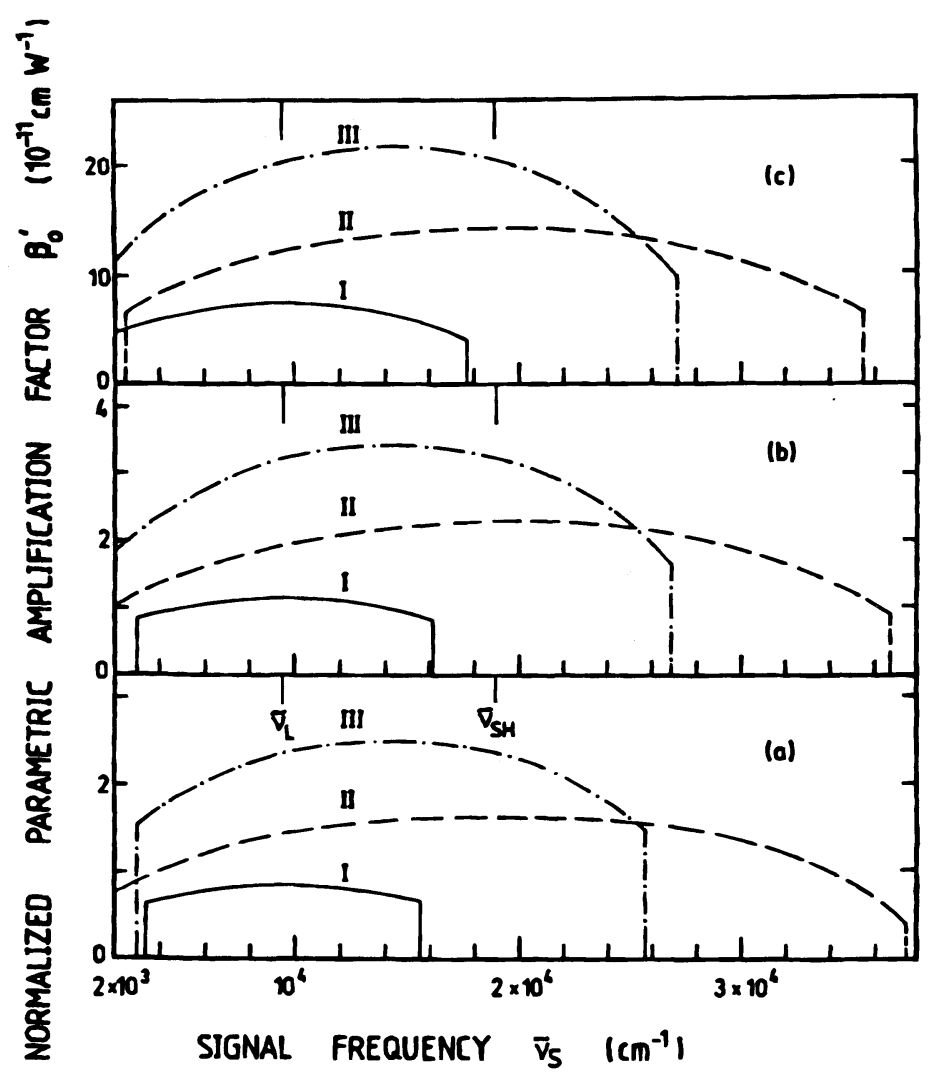

Figure 8 Normalized parametric amplification factor $\beta_{0}^{\prime}=\beta_{0} /\left(I_{1} I_{2}\right)^{1 / 2}$ for the interaction process (I) $\omega_{\mathrm{L}}+$ $\omega_{\mathrm{L}} \rightarrow \omega_{\mathrm{S}}+\omega_{1}$ (solid curves), (II) $\omega_{\mathrm{SH}}+\omega_{\mathrm{SH}} \rightarrow \omega_{\mathrm{S}}+\omega_{\mathrm{I}}$ (dashed curves), and (III) $\omega_{\mathrm{L}}+\omega_{\mathrm{SH}} \rightarrow \omega_{\mathrm{S}}+\omega_{\mathrm{I}}$ (dashdotted curves). (a) $\mathrm{D}_{2} \mathrm{O}$; (b) fused silica; (c) SF10 glass.

If $\omega_{1}+\omega_{2}$ approaches an electronic transition, then $\chi_{x x x x}^{(3)}\left(-\omega_{\mathrm{S}} ; \omega_{1}, \omega_{2},-\omega_{\mathrm{I}}\right)$ is sumfrequency enhanced. At resonance $\left(\omega_{1}+\omega_{2}\right.$ equal to an electronic transition frequency) two-photon absorption occurs $[1,2,51]$, which reduces the pump pulse intensity. Some two-photon resonance enhancement of $\chi^{(3)}\left(-\omega_{\mathrm{S}} ; \omega_{\mathrm{SH}}, \omega_{\mathrm{SH}},-\omega_{\mathrm{I}}\right)$ is expected for the flint glass SF10, leading to larger $\beta_{0}^{\prime}$ values for the amplification process (II) $\left(\omega_{\mathrm{SH}}+\omega_{\mathrm{SH}} \rightarrow \omega_{\mathrm{S}}+\omega_{\mathrm{I}}\right)$ than shown in Fig. 8c.

The wavevector mismatch quotient $\left|\kappa_{\mathrm{SI}, z}\right|=\left|\Delta k_{z} / \Delta \tilde{\nu}_{\mathrm{S}}\right|$ (Equation 24a with Fig. 2b, and Equation 22c with Fig. 2f) is displayed in Fig. 9 for the three interaction processes I $\left(\omega_{\mathrm{L}}+\omega_{\mathrm{L}} \rightarrow \omega_{\mathrm{S}}+\omega_{\mathrm{I}}\right)$, II $\left(\omega_{\mathrm{SH}}+\omega_{\mathrm{SH}} \rightarrow \omega_{\mathrm{S}}+\omega_{\mathrm{I}}\right)$, and III $\left(\omega_{\mathrm{L}}+\omega_{\mathrm{SH}} \rightarrow \omega_{\mathrm{S}}+\omega_{\mathrm{I}}\right) . \kappa_{\mathrm{SI}, z}$ limits the spectral amplification width of broadband signal light to $\Delta \tilde{\nu}_{\mathrm{S}, \max }=\beta_{0} /\left|\kappa_{\mathrm{SI}, z}\right|$ (Equations 27). Small $\kappa_{\mathrm{SI}, z}$ values are necessary for short signal pulse durations since the spectral width of bandwidth-limited pulses is inversely proportional to the pulse duration.

The wavevector mismatch quotient $\left|\kappa_{12, z}\right|=\left|\Delta k_{z} / \Delta \tilde{\nu}\right|$ (Equation 25a with Fig. 2c, and Equation 25c with Fig. 2g) for the process III $\left(\omega_{\mathrm{L}}+\omega_{\mathrm{SH}} \rightarrow \omega_{\mathrm{S}}+\omega_{\mathrm{I}}\right)$ is included in Fig. 9 


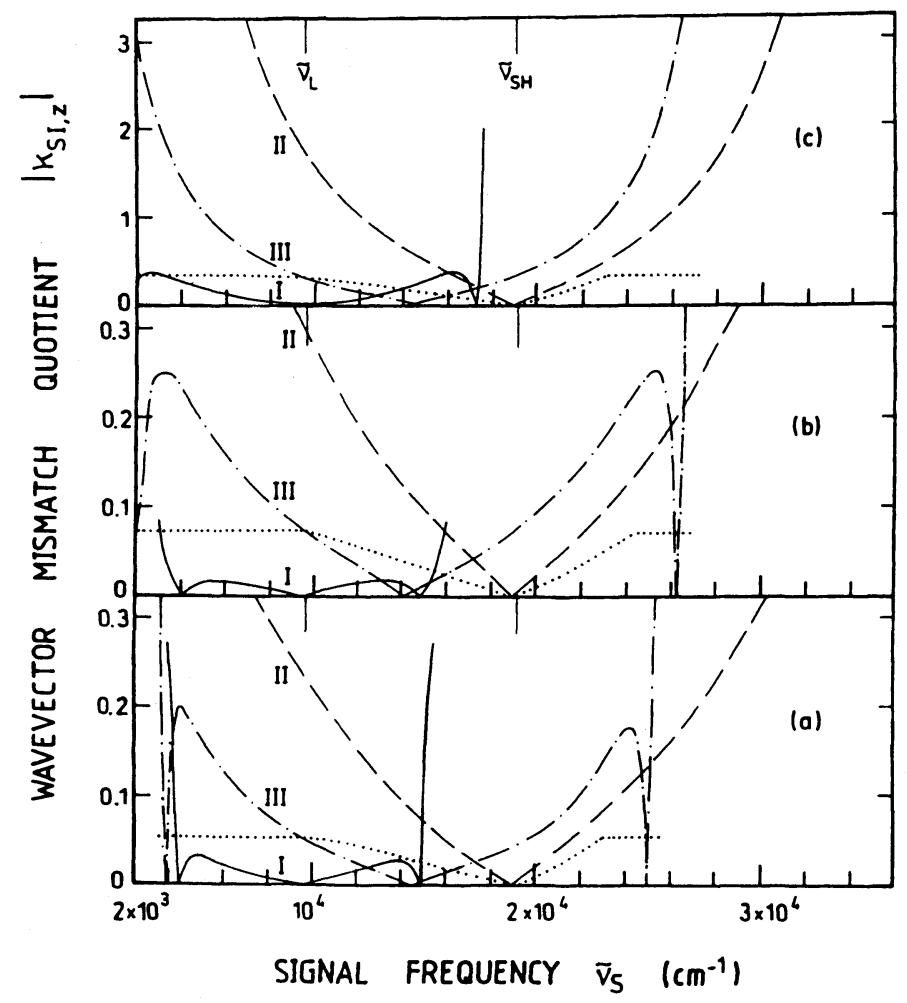

Figure 9 Wavevector mismatch quotients $\left|\kappa_{S \mid, z}\right|=\left|\Delta k_{z} / \Delta \tilde{\nu}_{S}\right|$ for the interaction processes (I) $\omega_{\mathrm{L}}+\omega_{\mathrm{L}} \rightarrow$ $\omega_{S}+\omega_{1}$ (solid curves), (II) $\omega_{S H}+\omega_{S H} \rightarrow \omega_{S}+\omega_{I}$ (dashed curves), and (III) $\omega_{\mathrm{L}}+\omega_{\mathrm{SH}} \rightarrow \omega_{\mathrm{S}}+\omega_{1}$ (dashdotted curves). The dotted curves represent the wavevector mismatch quotients $\left|\kappa_{12, z}\right|=\left|\Delta k_{z} / \Delta \tilde{\nu}\right|$ for the interaction process (III). (a) $\mathrm{D}_{2} \mathrm{O}$; (b) fused silica; (c) SF10 glass.

(dotted curves). For the pump-pulse degenerate processes I and II $\left|\kappa_{12,2}\right|$ is negligibly small (of the order of $10^{-7}$ ). Concerning efficient parametric amplification, $\kappa_{12, z}$ restricts the spectral pulse width to $\Delta \tilde{\nu}<\beta_{0} /\left|\kappa_{12, z}\right|$ and the pulse duration of bandwidth-limited pulses to $\Delta t>\gamma c_{0}\left|\kappa_{12, z}\right| / \beta_{0}$ with $\gamma=0.441$ for temporal Gaussian pulse shapes [52].

The wavevector mismatch quotients $\left|\kappa_{\mathrm{SI}, x}\right|=\left|\Delta k_{x} / \Delta \tilde{\nu}_{\mathrm{S}}\right|$ (Equation 24b with Fig. 2b, and Equation 24d with Fig. 2f) and $\left|\kappa_{12, x}\right|=\left|\Delta k_{x} / \Delta \tilde{\nu}\right|$ (Equation 25b with Fig. 2c, and Equation 25d with Fig. 2g) are displayed in Fig. 10.

\section{Limiting processes}

Concurrently with parametric four-photon amplification there occur other nonlinear optical effects. Self-phase modulation $[1,53]$ causes a chirp of the pump pulse frequencies versus time and results in an overall spectral pump pulse broadening. Cross-phase modulation induces a frequency chirp to the signal pulse [10, 54] (refractive index change is caused by pump pulses). Stimulated Raman scattering $\omega_{i} \rightarrow \omega_{\mathrm{R}}+\omega_{\mathrm{v}}(i=\mathrm{L}$ or SH) generates Stokes-shifted light at frequency $\omega_{\mathrm{R}}$ and may deplete the pump pulse intensity [1,47]. The parametric gain factor $\beta_{0}$ increases linearly with the effective pump pulse intensity $\left(I_{1} I_{2}\right)^{1 / 2}$. But there is an upper intensity limit $I_{\mathrm{d}, \text { th }}$ set by material damage in solids [55] 


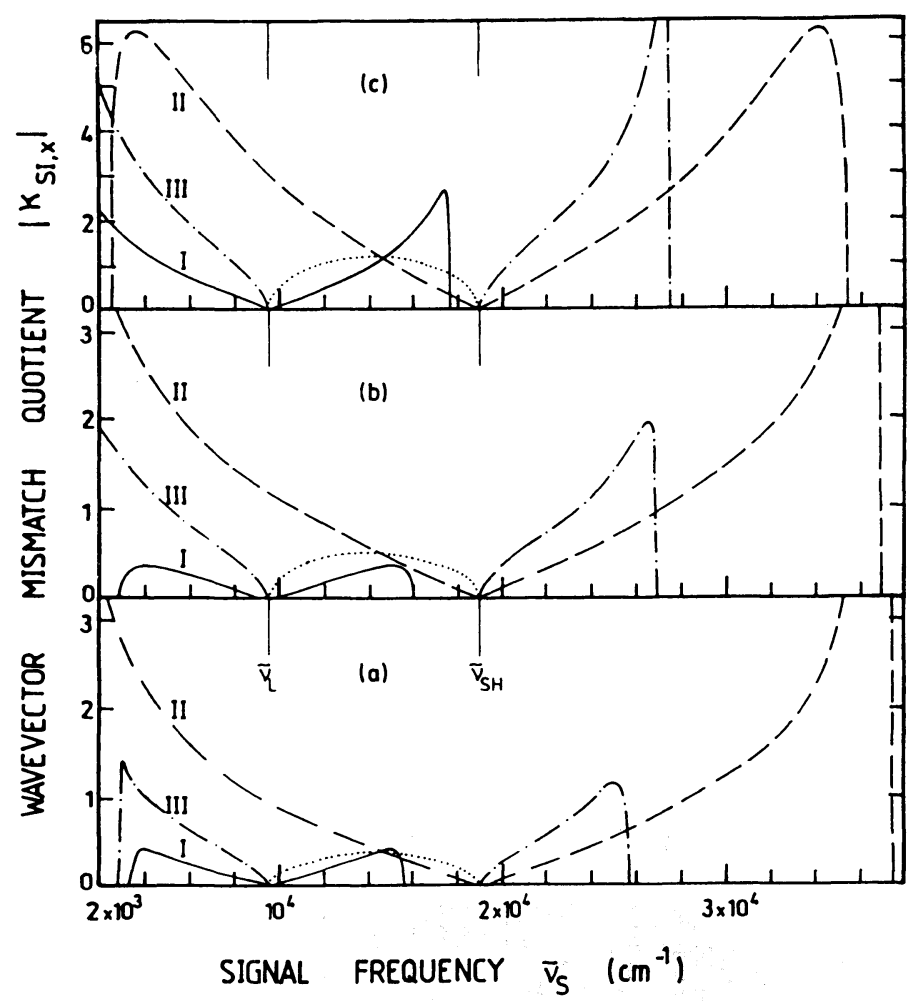

Figure 10 Wavevector mismatch quotients $\left|\kappa_{\mathrm{SI}, x}\right|=\left|\Delta k_{x} / \Delta \tilde{\nu}_{\mathrm{S}}\right|$ and $\left|\kappa_{12, x}\right|=\left|\Delta k_{x} / \Delta \tilde{\nu}\right|$ (dotted curve). Legend of Fig. 9 applies.

and dielectric breakdown in liquids [56]. At high pump pulse intensities and long sample lengths, self-focusing may occur, which increases the laser intensity in an uncontrolled way. The transverse phase mismatch $\Delta k_{x} d_{\text {eff }}$ increases linearly with the effective beam diameter $d_{\mathrm{eff}}=\tan (\varphi) l_{\mathrm{eff}} . \Delta k_{x} d_{\mathrm{eff}}$ should be less than $\pi$ to avoid reduction of parametric amplification [15, 37]. The limiting effects of these various processes on parametric fourphoton amplification are discussed in the following.

\subsection{Optical breakdown}

The maximum applicable pump pulse peak intensity is limited by the laser-induced optical damage threshold intensity $I_{\mathrm{d} \text {,th }}$. In Fig. 11 the surface damage threshold intensities of the glasses and the dielectric breakdown threshold intensity of $\mathrm{D}_{2} \mathrm{O}$ are plotted versus pulse duration. A wavelength dependence of $I_{\mathrm{d} \text {,th }}$ is neglected, i.e. $I_{\mathrm{d}, \mathrm{th}}\left(\lambda_{\mathrm{L}}\right)=I_{\mathrm{d}, \mathrm{th}}\left(\lambda_{\mathrm{SH}}\right)$ is assumed. Data are taken from [57] for the Schott glass SF10, from [58, 59] for fused silica, and from [56] for $\mathrm{D}_{2} \mathrm{O}$. An inverse square root dependence of $I_{\mathrm{d}, \text { th }}$ on pulse duration $\Delta t$ is assumed, i.e. $I_{\mathrm{d}, \mathrm{th}} \propto \Delta t^{-1 / 2}[58,59]$.

For a fixed pump pulse energy, the four-photon parametric gain is highest if the intensity is increased at the cost of the beam diameter, since the intensity rises proportionally to $d^{-2}$ while the overlap length $l_{\text {ov }}$ decreases only proportionally to $d$, so that the gain $G_{0}$ 


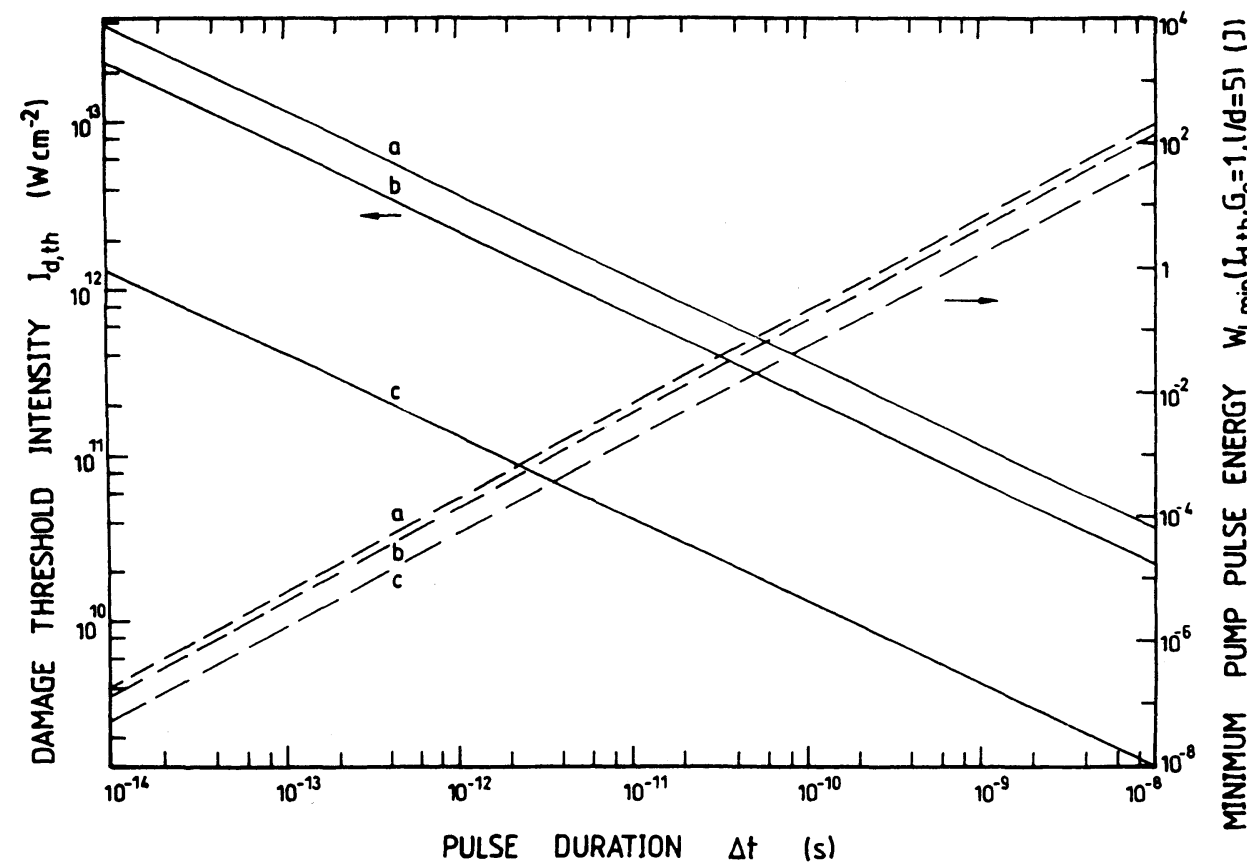

Figure 11 Damage threshold intensities $I_{\mathrm{d}, \text { th }}$ (solid curves), and minimum pump pulse energies $W_{\min }$ for parametric gain $G_{0}=1$ using $I / d=5$, and $I_{L}=I_{d, t h}$. The interaction process $(I) \omega_{L}+\omega_{L} \rightarrow \omega_{S}+\omega_{I}$ is considered. Curves a: $\mathrm{D}_{2} \mathrm{O}$. Curves b: fused silica. Curves c: SF10 glass.

increases proportionally to $d^{-1}$. In order to obtain high parametric gain it is therefore advantageous to increase the pump pulse intensity to near the damage threshold intensity.

The minimum pump pulse energy $W_{\min }\left(G_{0}=1, l / d=5, I_{\mathrm{L}}=I_{\mathrm{d}, \mathrm{th}}\right)$ necessary for a parametric gain of $G_{0}=1$ and a sample length to beam diameter ratio of $l / d=5$ (see Fig. 6) is plotted versus pulse duration in Fig. 11 (dashed curves) for the interaction process I $\left(\omega_{\mathrm{L}}+\omega_{\mathrm{L}} \rightarrow \omega_{\mathrm{S}}+\omega_{\mathrm{I}}\right) . W_{\min }$ is obtained from the relation $W_{\min } \approx I_{\mathrm{d}, \mathrm{th}} \Delta t d_{\min }^{2}$, where $d_{\min }$ is calculated from $G_{0}=\beta_{0} l_{\min }=\beta_{0}(l / d) d_{\min }=\beta_{0}^{\prime} I_{\mathrm{d}, \mathrm{th}}(l / d) d_{\min }$. The normalized parametric gain factor $\beta_{0}^{\prime}$ is displayed in Fig. 8, and the damage threshold intensities $I_{\mathrm{d}, \text { th }}$ are displayed by the solid curves in Fig. 11. The dashed curves in Fig. 11 show that the minimum pump pulse energy $W_{\min }$ is proportional to $\Delta t^{3 / 2}$. For a pump pulse duration of $\Delta t=5 \mathrm{ps}$ the minimum pump pulse energies are $W_{\mathrm{L}, \min }\left(G_{0}=1, \mathrm{D}_{2} \mathrm{O}\right) \approx 2.2 \mathrm{~mJ}$, $W_{\mathrm{L}, \text { min }}\left(G_{0}=1, \mathrm{Si}_{2} \mathrm{O}\right) \approx 1.6 \mathrm{~mJ}$, and $W_{\mathrm{L}, \text { min }}\left(G_{0}=1, \mathrm{SF} 10\right) \approx 0.65 \mathrm{~mJ}$ for $l_{\text {min }} / d=5$ and $\beta_{0, \max }^{\prime}$ (in wide frequency regions around $\nu_{\mathrm{L}}, l_{\mathrm{ov}} / d$ is much larger than 5 , leading to lower $W_{\mathrm{L}, \min }$ energies). The minimum pump pulse energy rises proportionally to $G_{0}^{2}$, because $G_{0}$ is proportional to $l, l$ is proportional to $d$, and $W$ is proportional to $d^{2}$.

For the interaction processes II and III, the minimum pump pulse energies are approximately factors of $2^{2}$ and $4^{2}$ smaller than the displayed curves for process I, since the $\beta_{0}^{\prime}$ values are factors of 2 and 4 larger (see Fig. 8), respectively.

The maximum parametric gain factors $\beta_{0, \max }=\beta_{0, \max }^{\prime} I_{\mathrm{d}, \text { th }}$ versus pulse duration are shown by the solid curves in Fig. 12 for the interaction process I $\left(\omega_{\mathrm{L}}+\omega_{\mathrm{L}} \rightarrow \omega_{\mathrm{S}}+\omega_{\mathrm{I}}\right)$. $\beta_{0, \max }$ reduces proportionally to $\Delta t^{-1 / 2}$ with increasing pulse duration. 


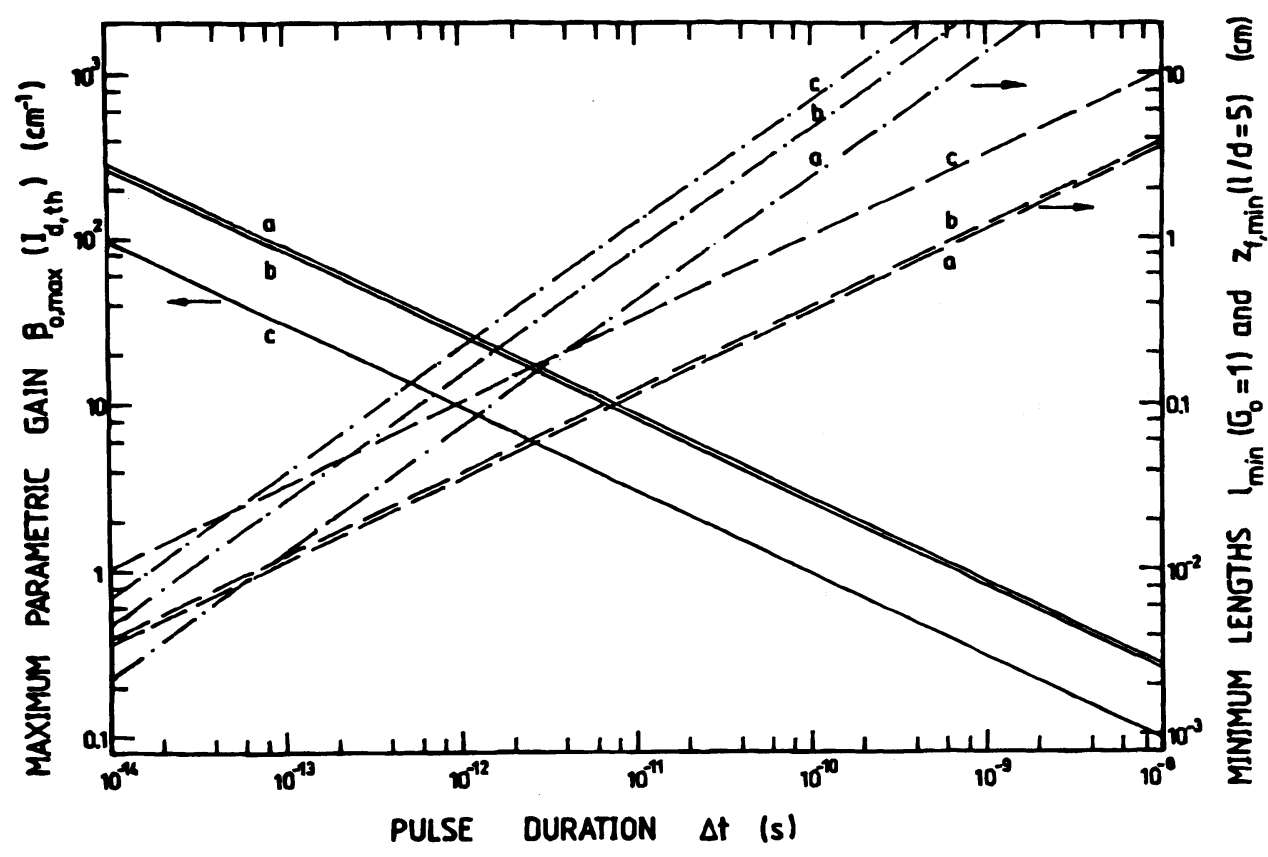

Figure 12 Maximum parametric gain factor $\beta_{0, \max }=\beta_{0}\left(\tilde{\nu}_{\mathrm{L}}, I_{\mathrm{d}, \mathrm{h}}\right)$ (solid curves), minimum sample length $I_{\min }\left(I_{\mathrm{d}, \mathrm{th}}, G_{0}=1\right)$ for $G_{0}=1$ (dashed curves), and minimum self-focusing length $z_{\mathrm{f}, \min }\left(I_{\mathrm{d}, \mathrm{th}}, / / d=5, G_{0}=1\right.$ ) (dash-dotted curves). The interaction process (I) $\left(\omega_{L}+\omega_{L} \rightarrow \omega_{S}+\omega_{I}\right)$ is considered. Curves a: $D_{2} O$. Curves b: fused silica. Curves c: SF10 glass.

The minimum sample lengths $l_{\min }$ necessary for $G_{0}=1$ at $I_{\mathrm{L}}=I_{\mathrm{d} \text {,th }}$ are shown by the dashed curves in Fig. 12 for the interaction process (I). $l_{\min }$ is obtained from the relation $G_{0}=\beta_{0, \max } l_{\min }$. The minimum sample length rises proportionally to $\Delta t^{1 / 2}$ with pulse duration. For a pulse duration of $\Delta t=5 \mathrm{ps}$ the minimum sample lengths are $l_{\min }\left(G_{0}=1\right.$, $\left.\mathrm{D}_{2} \mathrm{O}\right)=0.8 \mathrm{~mm}, l_{\min }\left(G_{0}=1, \mathrm{SiO}_{2}\right)=0.9 \mathrm{~mm}$, and $l_{\min }\left(G_{0}=1, \mathrm{SF} 10\right)=2.2 \mathrm{~mm}$.

\subsection{Optical absorption}

Whenever $l_{\min }\left(\nu_{\mathrm{S}}, G_{0}, I_{\mathrm{d}, \text { th }}\right)=G_{0} / \beta_{0}\left(\nu_{\mathrm{S}}, I_{\mathrm{d}, \text { th }}\right)$ becomes larger than the absorption length $l_{\alpha}$ (Equation 19), the absorption process limits the parametric amplification. The curves in Fig. 13 show border curves $\tilde{\nu}_{\mathrm{S}, \mathrm{b}}$ of parametric amplification caused either by signal absorption (case of Equation 19a) or by noncollinear phase-matching limitation (horizontal lines, Fig. 5). The bottom abscissa refers to $G_{0}=10$ and the top abscissa to $G_{0}=1$. The pump pulse intensities are set to $I_{\mathrm{L}}=I_{\mathrm{d} \text {,th }}$ (process I), $I_{\mathrm{SH}}=I_{\mathrm{d} \text {,th }}$ (process II), and $I_{\mathrm{L}}=I_{\mathrm{SH}}=I_{\mathrm{d}, \mathrm{th}} / 2$ (process III). For $G_{0}=1$ a spectral limitation of parametric amplification by signal and idler absorption is small up to pulse durations of a few nanoseconds. For $G_{0}=10$ the influence of absorption is weak up to a few tens of picoseconds.

\subsection{Self-focusing}

Self-focusing [1] of spatially bell-shaped pump pulses of frequency $\omega_{i}(i=\mathrm{L}$ or $\mathrm{SH}$ ) is caused by an intensity-dependent refractive index contribution, $\Delta n_{i}=n_{2, \mathrm{nl}}\left|E_{i}\right|^{2} / 2=\gamma_{2} I_{i}$, 


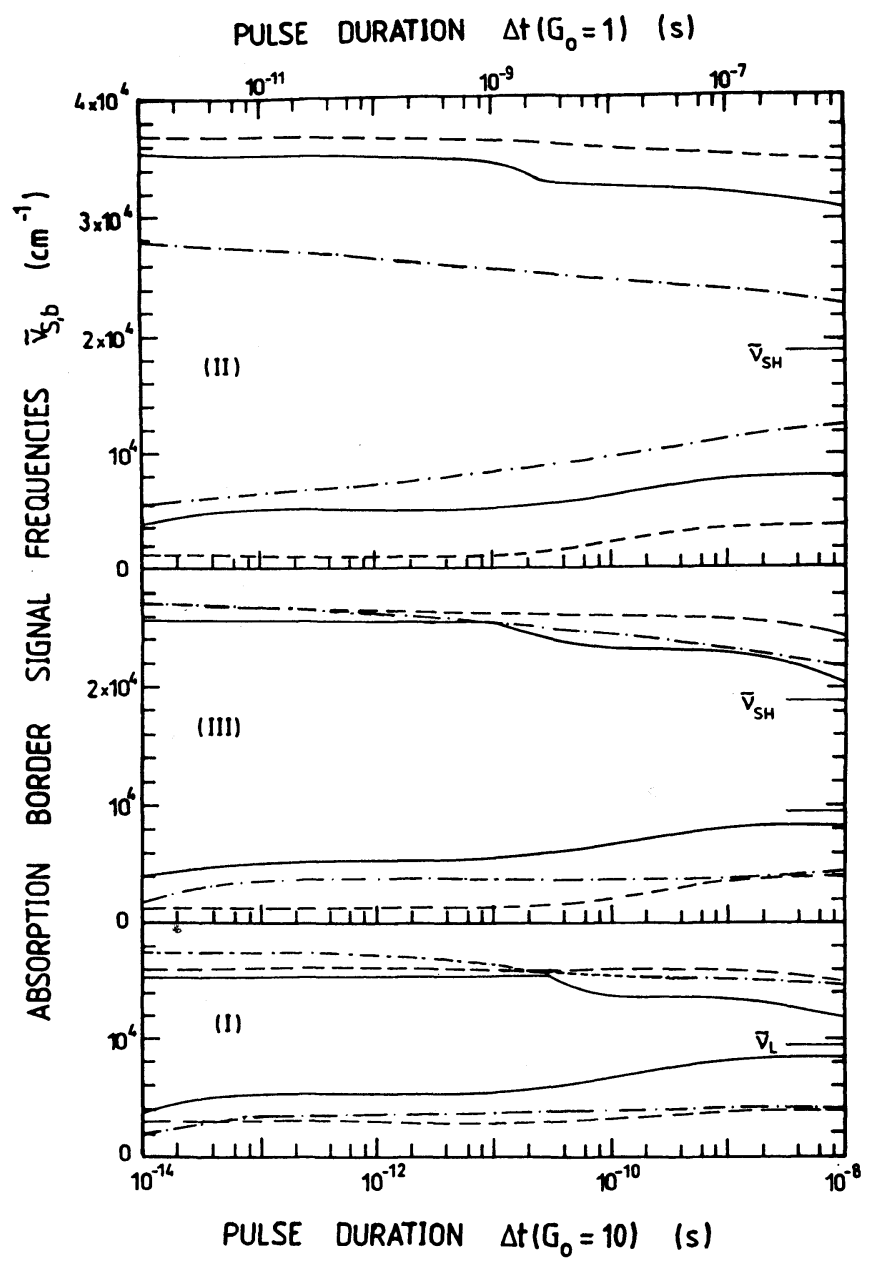

Figure 13 Signal frequency borders $\tilde{\nu}_{\mathrm{S}, \mathrm{b}}$ caused by signal absorption and noncollinear phase-matching limits. The small parametric gain situation is considered (Equation 19a). Upper abscissa applies to $G_{0}=1$, $\beta_{0}\left(\tilde{\nu}_{\mathrm{S}, \mathrm{b}}, I_{\mathrm{d}, \mathrm{th}}\right)$ and $I=I_{\min }\left(G_{0}=1\right)$; lower abscissa applies to $G_{0}=10, \beta_{0}\left(\tilde{\nu}_{\mathrm{S}, \mathrm{b}}, I_{\mathrm{d}, \mathrm{th}}\right)$ and $I_{\min }\left(G_{0}=10\right)$. Solid curves, $D_{2} O$. Dashed curves, fused silica (Infrasil). Dashed-dotted curves, SF10 glass. (I): interaction process (I) $\left(\omega_{\mathrm{L}}+\omega_{\mathrm{L}} \rightarrow \omega_{\mathrm{S}}+\omega_{\mathrm{I}}, I_{\mathrm{L}}=I_{\mathrm{d}, \mathrm{th}}\right)$. (III): interaction process (III) $\left(\omega_{\mathrm{L}}+\omega_{\mathrm{SH}} \rightarrow \omega_{\mathrm{S}}+\omega_{\mathrm{I}}, I_{\mathrm{L}}=I_{\mathrm{SH}}=\right.$ $\left.I_{\mathrm{d}, \mathrm{th}} / 2\right)$. (II): interaction process (II) $\left(\omega_{\mathrm{SH}}+\omega_{\mathrm{SH}} \rightarrow \omega_{\mathrm{S}}+\omega_{\mathrm{I}}, I_{\mathrm{SH}}=I_{\mathrm{d}, \mathrm{th}}\right)$.

to the linear refractive index $n_{i}$, i.e. $n_{i}\left(I_{i}\right)=n_{i}+\Delta n_{i}$. The relation between the electric field coefficient $n_{2, \mathrm{n}}$, the intensity coefficient $\gamma_{2}$, and the real part of the nonlinear susceptibility $\chi_{x x x x}^{(3)^{\prime}}\left(-\omega_{i} ; \omega_{i},-\omega_{i}, \omega_{i}\right)=\chi_{\mathrm{nl}}^{(3)^{\prime}}$ is given by [60]

$$
n_{2, \mathrm{nl}}=n_{i} \epsilon_{0} c_{0} \gamma_{2}=\frac{3}{n_{i}} \chi_{\mathrm{nl}}^{(3)^{\prime}}
$$

The nonlinear susceptibility $\chi_{x x x x}^{(3)}\left(-\omega_{i} ; \omega_{i},-\omega_{i}, \omega_{i}\right)$ is composed of a nonresonant electronic contribution (equal to $\chi_{\mathrm{P}}^{(3)}$ used in Equation 7), a zero-frequency resonant part [61], 
TABLE II Nonlinear optical parameters

\begin{tabular}{lllll}
\hline Parameter & $\mathrm{D}_{2} \mathrm{O}$ & $\mathrm{SiO}_{2}$ & $\mathrm{SF} 10$ & Comments \\
\hline$\chi_{\mathrm{P}}^{(3)}\left(\mathrm{m}^{2} \mathrm{~V}^{-2}\right)$ & $2.2 \times 10^{-23}$ & $3.6 \times 10^{-23}$ & $3.2 \times 10^{-22}$ & {$[44]$} \\
$\chi_{\mathrm{nl}}^{(3)^{\prime}}\left(\mathrm{m}^{2} \mathrm{~V}^{-2}\right)$ & $9.8 \times 10^{-23}$ & $5.5 \times 10^{-23}$ & $\sim 6 \times 10^{-21}$ & Equation 29 \\
$\gamma_{2}\left(\mathrm{~m}^{2} \mathrm{~W}^{-1}\right)$ & $6.3 \times 10^{-20}[56]$ & $3.2 \times 10^{-20}[67]$ & $\sim 2 \times 10^{-18}[57]$ & \\
$\chi_{\mathrm{R}}^{(3)^{\prime \prime}}\left(\mathrm{m}^{2} \mathrm{~V}^{-2}\right)$ & $3.9 \times 10^{-23}$ & $1.5 \times 10^{-23}$ & $\sim 1.4 \times 10^{-22}$ & Equation 35 \\
$g_{\mathrm{R}}\left(\mathrm{m} \mathrm{W}^{-1}\right)$ & $4.6 \times 10^{-13}[68]^{\mathrm{a}}$ & $1.8 \times 10^{-13}[64]$ & $\sim 1.2 \times 10^{-12 \mathrm{~b}}$ & $\lambda_{\mathrm{L}}=1054 \mathrm{~nm}$ \\
$G_{\mathrm{R}}\left[I_{\mathrm{d}, \mathrm{h}}, l_{\min }\left(G_{0}=1\right)\right]$ & 1.25 & 0.31 & 1.6 & Equation 36 \\
$\nu_{\mathrm{d}}$ & 53 & 74 & 30 & From Fig. 3 \\
$f\left(\nu_{\mathrm{L}}, \Delta t=5 \mathrm{ps}\right)$ & 0.71 & 2.9 & $\sim 0.05$ & Equation 37 \\
\hline
\end{tabular}

${ }^{a}$ Value for $\mathrm{H}_{2} \mathrm{O}$ : the same value is assumed for $\mathrm{D}_{2} \mathrm{O}$.

${ }^{\mathrm{b}}$ Estimated from Raman parameters presented in [69] for SF6 and SF7 glasses.

The conversion between SI units (used here) and e.s.u. units of the third-order nonlinear susceptibilities is $\chi^{(3)}($ e.s.u. $)=\left[9 \times 10^{8} /(4 \pi)\right] \chi^{(3)}(\mathrm{SI})[80]$.

and a two-photon resonant term if $2 \omega_{i}$ is near to an electronic transition frequency. $\chi_{\mathrm{nl}}^{(3)^{\prime}}$ values of the investigated media are listed in Table II. $\chi_{\mathrm{nl}}^{(3)}\left(\lambda_{\mathrm{L}}=1054 \mathrm{~nm}\right)$ and $\chi_{\mathrm{nl}}^{(3)}\left(\lambda_{\mathrm{SH}}=527 \mathrm{~nm}\right)$ are assumed to be equal.

The self-focusing length $z_{\mathrm{f}}$ of a Gaussian pulse of beam diameter $d$ (FWHM) is given by $[1,62]$

$$
z_{\mathrm{f}}=\frac{[2 \ln (2)]^{-1} \pi n_{i} \tilde{\nu}_{i} d^{2}}{\left[2 \pi^{2}(\ln 2)^{-1} n_{i} \gamma_{2} \tilde{\nu}_{i}^{2} d^{2} I_{i}-1\right]^{1 / 2}+\theta}
$$

where $\theta$ is the divergence angle of the incident laser beam $(\theta=0$ in our calculations). The dash-dotted lines in Fig. 12 represent the minimum self-focusing lengths $z_{\mathrm{f}, \min }\left(G_{0}=1\right.$, $l / d=5$ ) versus pulse duration for pulse intensities at the damage threshold $I_{\mathrm{d} \text {,th }}$ and minimum beam diameter $d_{\min }$ necessary for $G_{0}=1$ in the case of $l / d=5$ (corresponding to $W_{\min }$ of Fig. 11). The self-focusing length $z_{\mathrm{f} \text {, min }}$ increases proportionally to $\Delta t^{3 / 4}$ with pulse duration. Down to the subpicosecond range, the minimum self-focusing length $z_{\mathrm{f}, \min }\left(G_{0}=1, l / d=5\right)$ is longer than the minimum sample length $l_{\min }\left(G_{0}=1\right)$. Whenever self-focusing might be a problem it can be avoided by increasing the laser beam diameter $d$ since $z_{\mathrm{f}}$ is approximately proportional to $d$ (Equation 30 ). In this case the necessary pump pulse energy would increase quadratically.

\subsection{Self-phase modulation and cross-phase modulation}

The intensity-dependent refractive index contribution $\Delta n_{i}=\gamma_{2} I_{i}(i=\mathrm{L}$ or SH$)$ causes selfphase modulation of temporally bell-shaped pump pulses of frequency $\omega_{i}[1,14,53]$. The carrier frequency $\tilde{\nu}_{\mathrm{c}}$ of the pulses becomes chirped according to [62,63]

$$
\tilde{\nu}_{\mathrm{c}}(t)=-\frac{\tilde{\nu}_{i}}{c_{0}} \gamma_{2} l \frac{\partial I_{i}(t)}{\partial t}
$$

The spectral broadening $\Delta \tilde{\nu}_{\mathrm{SPM}}$ of a Gaussian pulse of peak intensity $I_{0, i}$ is approximately given by $[53,62]$

$$
\Delta \tilde{\nu}_{\mathrm{SPM}}=\frac{4[2 \ln (2)]^{1 / 2} \exp (-1 / 2) \tilde{\nu}_{i} \gamma_{2} I_{0, i} l}{c_{0} \Delta t}
$$




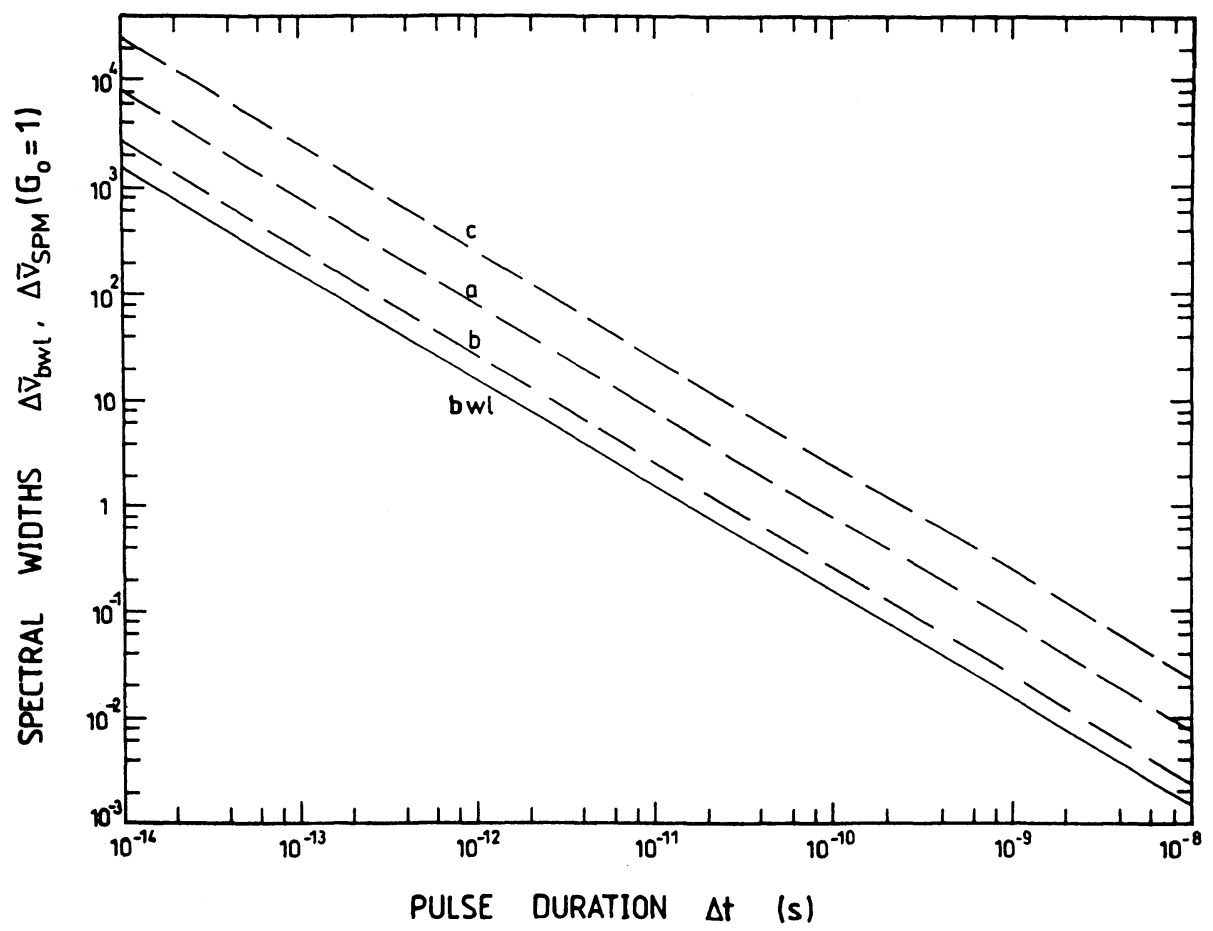

Figure 14 Spectral width (FWHM) of bandwidth-limited Gaussian pulses (solid curve) and minimum selfphase modulation width $\Delta \tilde{\nu}_{\mathrm{SPM}, \min }\left(I_{\mathrm{d}, \mathrm{th}}, G_{0}=1, I_{\min }\right)$ of pulses of temporal Gaussian shape. The interaction process (I) $\omega_{L}+\omega_{L} \rightarrow \omega_{S}+\omega_{I}$ is considered. Curve a: $D_{2} O$. Curve b: fused silica. Curve c: SF10 glass.

In Fig. $14 \Delta \tilde{\nu}_{\mathrm{SPM}}$ versus $\Delta t$ is displayed for the case of $\lambda_{\mathrm{L}}=1054 \mathrm{~nm}, I_{\mathrm{L}}=I_{\mathrm{d} \text {,th }}$ and $G_{0}=1$ (dashed curves). The solid curve in Fig. 14 shows the spectral width $\Delta \tilde{\nu}_{\text {bwl }}$ of bandwidth-limited Gaussian pulses. For all investigated substances it is $\Delta \tilde{\nu}_{\mathrm{SPM}}\left(G_{0}=1\right)>\Delta \tilde{\nu}_{\mathrm{bwl}} . \Delta \tilde{\nu}_{\mathrm{SPM}}$ rises linearly with $G_{0}$ (both quantities are proportional to $\left.I_{0, i} l\right)$.

The frequency chirp (parallel shift of the frequencies $\omega_{1}$ and $\omega_{2}$ in the interaction $\left.\omega_{1}+\omega_{2} \rightarrow \omega_{\mathrm{S}}+\omega_{\mathrm{I}}\right)$ causes a temporally chirping wavevector mismatch of modulation width $\Delta k_{\mathrm{SPM}}=\left|\kappa_{\mathrm{SPM}, z}\right| \Delta \tilde{\nu}_{\mathrm{SPM}}$ (Equations 26a and c). Parametric four-photon amplification is limited to regions where $\beta_{0}>\Delta k_{\mathrm{SPM}, z}$ (Equation 27c).

The wavevector mismatch coefficient $\left|\kappa_{\mathrm{SPM}, z}\right|=\left|\Delta k_{z}\right| / \Delta \tilde{\nu}_{\mathrm{SPM}}$ versus signal frequency $\tilde{\nu}_{\mathrm{S}}$ is displayed in Fig. 15 for the three interaction processes I, II, III and the three studied substances $\mathrm{D}_{2} \mathrm{O}, \mathrm{SiO}_{2}$ and $\mathrm{SF} 10$. The curves were obtained by use of Equations $26 \mathrm{a}$ (Fig. 2d) and 26c (Fig. 2h). $\left|\kappa_{\mathrm{SPM}, z}\right|$ is of the same magnitude as $\left|\kappa_{\mathrm{SI}, z}\right|$ (Fig. 9).

The wavevector mismatch due to self-phase modulation limits the regions of possible parametric four-photon amplification. Border curves $\tilde{\nu}_{\mathrm{S}, \mathrm{b}}(\Delta t)$ are presented in Fig. 16. The top abscissa applies to $G_{0}=1, \beta_{0}=\beta_{0}\left(\tilde{\nu}_{\mathrm{S}, \mathrm{b}}, I_{\mathrm{d}, \mathrm{th}}\right)$ and $l=l_{\min }\left(\tilde{\nu}_{\mathrm{S}, \mathrm{b}}, G_{0}=1\right)$, while the bottom abscissa applies to $G_{0}=10, \beta_{0}\left(\tilde{\nu}_{\mathrm{S}, \mathrm{b}}, I_{\mathrm{d}, \mathrm{th}}\right)$ and $l_{\min }\left(\tilde{\nu}_{\mathrm{S}, \mathrm{b}}, G_{0}=10\right)$. The curves are obtained by using the relation $\Delta k_{\mathrm{SPM}, z}=\beta_{0}\left(\tilde{\nu}_{\mathrm{S}, \mathrm{b}}, I_{\mathrm{d}, \mathrm{th}}\right)=\left|\kappa_{\mathrm{SPM}, z}\right| \Delta \tilde{\nu}_{\mathrm{SPM}}\left(G_{0}\right)$ (for the interaction process III, $\beta_{0}\left(\tilde{\nu}_{\mathrm{S}, \mathrm{b}}, I_{\mathrm{d}, \mathrm{th}} / 2\right)$ is used $)$. 


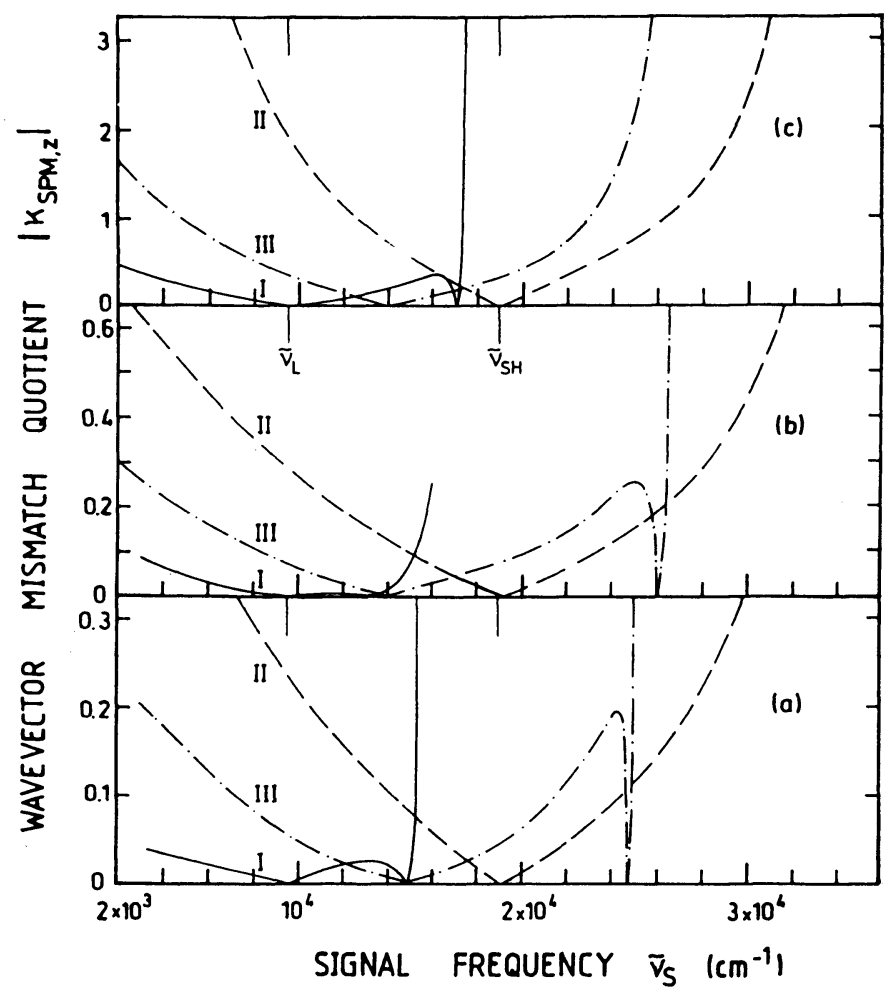

Figure 15 Wavevector mismatch quotients $\left|\kappa_{\mathrm{SPM}, z}\right|=\left|\Delta k_{z} / \Delta \tilde{\nu}_{\mathrm{SPM}}\right|$ due to self-phase modulation for the interaction processes (I) $\left(\omega_{\mathrm{L}}+\omega_{\mathrm{L}} \rightarrow \omega_{\mathrm{S}}+\omega_{1}\right.$, solid curves); process (II) ( $\omega_{S H}+\omega_{S H} \rightarrow \omega_{S}+\omega_{1}$, dashed curves); and process (III) $\left(\omega_{\mathrm{L}}+\omega_{\mathrm{SH}} \rightarrow \omega_{\mathrm{S}}+\omega_{1}\right.$, dash-dotted curves). (a) $\mathrm{D}_{2} \mathrm{O}$; (b) fused silica; (c) SF10 glass.

In the case of $G_{0}=1\left(G_{0}=10\right)$ the self-phase modulation practically does not restrict the spectral range of possible parametric amplification for $\mathrm{D}_{2} \mathrm{O}$ and fused silica when $\Delta t \gtrsim 0.1 \mathrm{ps}(\Delta t \gtrsim 10 \mathrm{ps})$, while for the flint glass SF10 the spectral range is strongly reduced up to $\Delta t \approx 1 \mathrm{~ns}(\Delta t \approx 100 \mathrm{~ns})$. At a fixed pulse duration the frequency range of feasible gain $G_{0}$ narrows with increasing $G_{0}$, since larger $G_{0}$ values require longer sample lengths $l$ and the spectral broadening by self-phase modulation is proportional to $l\left[\Delta \tilde{\nu}_{\mathrm{SPM}}\left(G_{0}=10\right)=10 \Delta \tilde{\nu}_{\mathrm{SPM}}\left(G_{0}=1\right)\right]$, while $\beta_{0}$ is independent of $l$ (Equation 5).

The vertical lines in Fig. 16 (upper lines: $G_{0}=1$; lower lines: $G_{0}=10$ ) indicate the time positions where the minimum spectral broadening due to self-phase modulation $\Delta \tilde{\nu}_{\mathrm{SPM}}\left(I=I_{\mathrm{d}, \mathrm{th}}, l=l_{\text {min }}\right)$ is equal to the spectral parametric amplification width. For shorter pulse durations the efficiency of parametric amplification is reduced.

The wavevector mismatch coefficient $\left|\kappa_{\text {SPM }, x}\right|=\left|\Delta k_{x}\right| / \Delta \tilde{\nu}_{\text {SPM }}$ versus signal frequency $\tilde{\nu}_{\mathrm{S}}$ is displayed in Fig. 17 for the three interaction processes I, II, III and the substances $\mathrm{D}_{2} \mathrm{O}$, $\mathrm{SiO}_{2}$ and SF10. $\kappa_{\mathrm{SPM}, x}$ is of the same order of magnitude as $\kappa_{\mathrm{SI}, x}$ and $\kappa_{12, x}$.

A comparison of the curves in Figs 17, 15 and 5 indicates that $\left|\kappa_{\mathrm{SPM}, x}\right| \tan (\varphi)<\left|\kappa_{\mathrm{SPM}, z}\right|$. Within the borders $\tilde{\nu}_{\mathrm{S}, \mathrm{b}}$ of Fig. 16 the transversal phase mismatch is $\Delta k_{x} d_{\mathrm{eff}}=$ $\left|\kappa_{\mathrm{SPM}, x}\right| \Delta \tilde{\nu}_{\mathrm{SPM}}\left(G_{0}\right) \tan (\varphi) l_{\min }\left(G_{0}\right)<\left|\kappa_{\mathrm{SPM}, z}\right| \Delta \tilde{\nu}_{\mathrm{SPM}}\left(G_{0}\right) l_{\min }\left(G_{0}\right)<G_{0}$. For $G_{0}<10$ the 


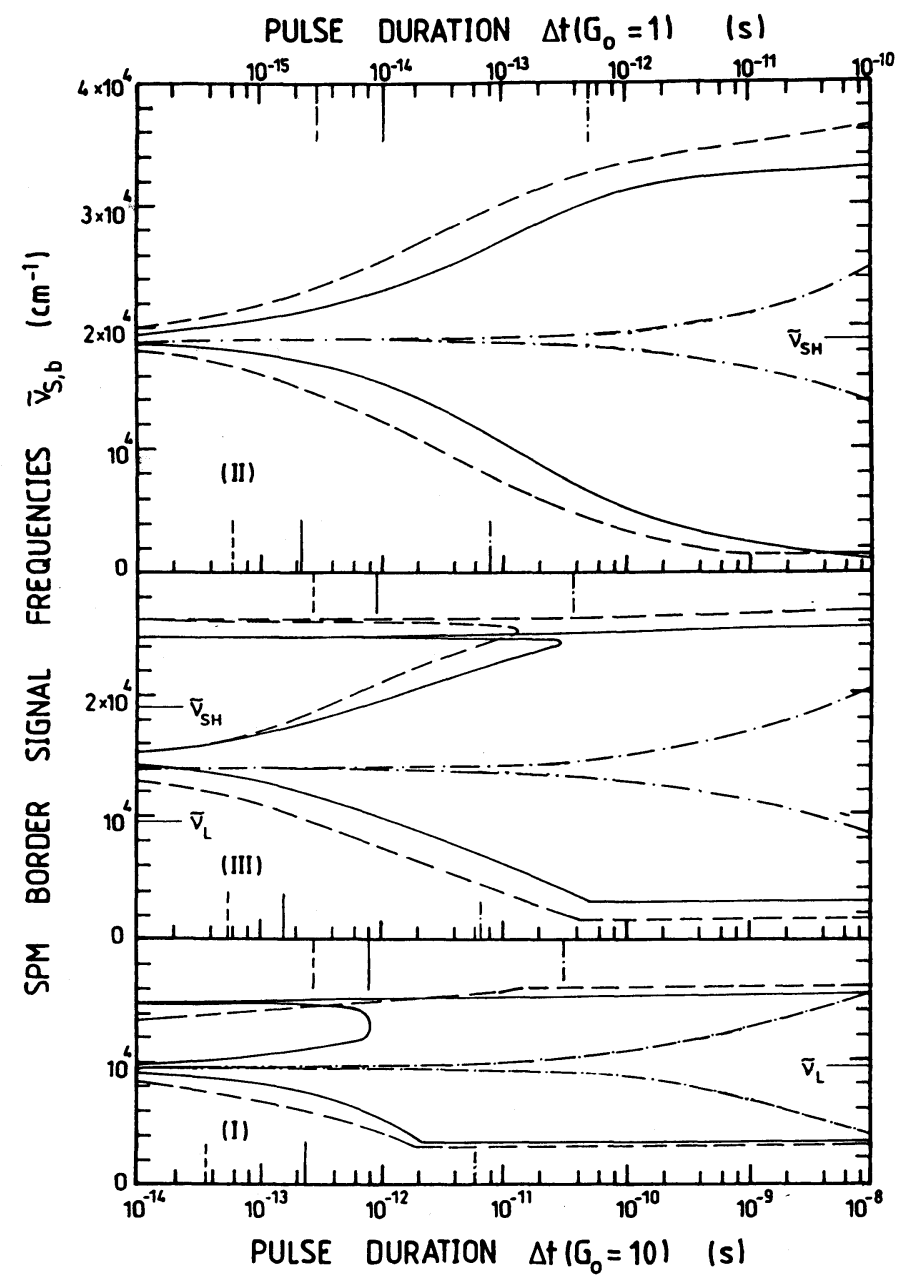

Figure 16 Signal frequency borders $\tilde{\nu}_{\mathrm{S}, \mathrm{b}}$ caused by self-phase modulation and noncollinear phase-matching limits. Parametric gain is $G_{0}=1$ for upper abscissa and $G_{0}=10$ for lower abscissa. The vertical lines mark the pulse duration positions where the spectral width between the borders is equal to the spectral self-phase modulation width. Otherwise the legend of Fig. 13 applies.

requirement $\Delta k_{x} d_{\mathrm{eff}}<\pi$ is fulfilled and the influence of transversal phase mismatch may be neglected.

The pump pulse propagation through the medium modifies the refractive index at the signal frequency $\nu_{\mathrm{S}}$ according to $\Delta n_{\mathrm{S}}=2 \gamma_{2} I_{i}(i=\mathrm{L}$ or SH) (the factor of 2 arises because of the lifting of frequency degeneracy) $[14,54]$. This refractive index change causes a signal frequency chirping and a signal frequency broadening of twice the magnitude of the pump pulse chirping and broadening. This phenomenon is called cross-phase modulation, XPM [54]. Since the cross-phase modulation is twice as strong as the self-phase modulation, the resulting wavevector mismatch $\Delta k_{\text {tot }}$ including cross-phase modulation $\Delta k_{\mathrm{XPM}}$ and selfphase modulation $\Delta k_{\mathrm{SPM}}$ is approximately the same as $\Delta k_{\mathrm{SPM}}$ considered above where 


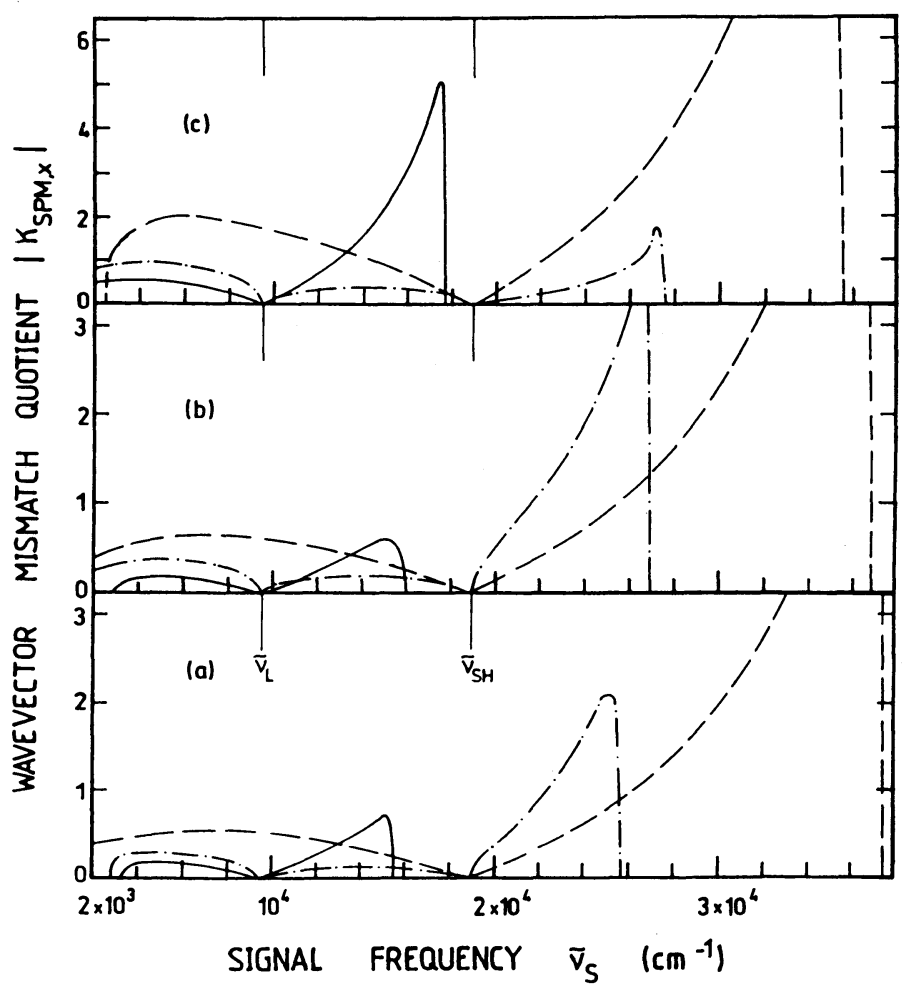

Figure 17 Wavevector mismatch quotients $\left|\kappa_{\mathrm{SPM}, x}\right|=\left|\Delta k_{x} / \Delta \tilde{\nu}_{\mathrm{SPM}}\right|$. Legend of Fig. 15 applies.

$\Delta \nu_{\mathrm{XPM}}$ was not included $\left(\Delta k_{\mathrm{tot}} \approx \Delta k_{\mathrm{XPM}}-\Delta k_{\mathrm{SPM}}=\Delta k_{\mathrm{SPM}}\right)$. Therefore the curves presented in Figs 15 to 17 apply as well to the combined effect of self-phase modulation and cross-phase modulation.

\subsection{Temporal spreading}

The temporal spreading between signal pulse and pump pulses would limit the efficiency of parametric amplification whenever $\delta t_{1, \mathrm{~S}} \gtrsim \Delta t$. The temporal spreading is proportional to the sample length. The minimum temporal spreading is given by $\delta t_{1, \mathrm{~S}, \min }=$ $\left|\delta t_{1, \mathrm{~S}} / l\right| l_{\min }\left(I_{\mathrm{d}, \mathrm{th}}, G_{0}, \nu_{\mathrm{S}}\right)$. Using the $\delta t_{1, \mathrm{~S}} / l$ curves of Fig. 7 and the $l_{\min }$ curves of Fig. 12, one finds that $\delta t_{1, \mathrm{~S}}$ remains less than $\Delta t$ in the parametric amplification regions of Fig. 16 determined by self-phase modulation.

\subsection{Stimulated Raman scattering}

The Stokes-Raman light generation by steady-state stimulated Raman scattering is given by [47]

$$
I_{\mathrm{R}}(l)=I_{\mathrm{R}, \mathrm{N}}\left[\exp \left(g_{\mathrm{R}} I_{\mathrm{L}} l\right)-1\right]
$$

where $I_{\mathrm{R}, \mathrm{N}}$ is the Stokes noise intensity, $g_{\mathrm{R}}$ is the steady-state Raman gain factor and $l$ is 
the sample length. $I_{\mathrm{R}, \mathrm{N}}$ is approximately given by [47]

$$
I_{\mathrm{R}, \mathrm{N}} \approx \frac{\pi \hbar \omega_{\mathrm{R}}^{3} n_{\mathrm{R}}^{2}}{2(2 \pi)^{3} c_{0}^{2}} \Delta \Omega \Delta \omega_{\mathrm{R}}
$$

where $\omega_{\mathrm{R}}$ is the angular Raman-Stokes frequency, $n_{\mathrm{R}}$ is the refractive index at the Raman frequency, $\Delta \Omega$ is the solid angle of efficient stimulated Raman emission, and $\Delta \omega_{R}$ is the angular Raman linewidth.

The steady-state Raman gain factor $g_{\mathrm{R}}$ is related to the imaginary part of the Raman third-order nonlinear susceptibility $\chi_{x x x x}^{(3)^{\prime \prime}}\left(-\omega_{\mathrm{R}} ; \omega_{i},-\omega_{i}, \omega_{\mathrm{R}}\right)=\chi_{\mathrm{R}}^{(3)^{\prime \prime}}$ by [47]

$$
g_{\mathrm{R}}=6 \frac{2 \omega_{\mathrm{R}}}{n_{i} n_{\mathrm{R}} \epsilon_{0} c_{0}^{2}} \chi_{\mathrm{R}}^{(3)^{\prime \prime}}
$$

Values of $g_{\mathrm{R}}$ at $\lambda_{\mathrm{L}}=1054 \mathrm{~nm}$ and of $\chi_{\mathrm{R}}^{(3)^{\prime \prime}}$ for the investigated media are listed in Table II.

Efficient stimulated Raman scattering causing pump pulse depletion occurs for [47, 64]

$$
G_{\mathrm{R}}=g_{\mathrm{R}} I_{\mathrm{L}} l \gtrsim 20=G_{\mathrm{R}, \mathrm{sat}}
$$

$G_{\mathrm{R}}$ is independent of pulse duration as long as a reduction of $g_{\mathrm{R}}$ at short pulse durations is neglected [47]. In Table II values of $G_{\mathrm{R}}$ are listed for $\lambda_{\mathrm{L}}=1054 \mathrm{~nm}, I_{\mathrm{L}}=I_{\mathrm{d} \text {,th }}$ and $l=l_{\min }\left(G_{0}=1\right)$. The listed $G_{\mathrm{R}}$ values are small compared to $G_{\mathrm{R}, \text { sat }} . G_{\mathrm{R}}$ increases linearly with $G_{0}$. Up to parametric gain factors of $G_{0}\left(\mathrm{D}_{2} \mathrm{O}\right) \approx 15, G_{0}\left(\mathrm{SiO}_{2}\right) \approx 60$ and $G_{0}(\mathrm{SF} 10) \approx 12$ the pump pulse intensity is not depleted by stimulated Raman scattering and stimulated Raman scattering does not affect the parametric four-photon amplification.

Stimulated Raman scattering is intrinsically phase-matched and occurs over the whole sample length, while efficient noncollinear phase-matched parametric four-photon amplification is limited to the effective interaction length $l_{\text {eff }}$ (Equation 23). In order to avoid enhanced stimulated Raman scattering, the sample length should be kept less than or equal to the frequency-dependent effective interaction length.

\section{Note added in proof}

In this paper noncollinear phase matched parametric four-photon amplification of nondivergent light beams was considered. For divergent beams the angular spreading causes wavevector mismatching and reduces the efficiency of parametric amplification (Equation 5). The influence of an angular deviation of the signal beam from the phase-matching angle $\varphi$ on the longitudinal phase-mismatch $\Delta k_{z}$ is shown in Fig. 18 for the the three processes (I) $\omega_{\mathrm{L}}+\omega_{\mathrm{L}} \rightarrow \omega_{\mathrm{S}}+\omega_{\mathrm{I}}$, (II) $\omega_{\mathrm{SH}}+\omega_{\mathrm{SH}} \rightarrow \omega_{\mathrm{S}}+\omega_{\mathrm{I}}$, and (III) $\omega_{\mathrm{L}}+\omega_{\mathrm{SH}} \rightarrow \omega_{\mathrm{S}}+\omega_{\mathrm{I}}$ and the three media (a) $\mathrm{D}_{2} \mathrm{O}$, (b) fused silica and (c) SF10 glass. $\mathrm{d}\left(\Delta k_{z}\right) / \mathrm{d} \varphi$ increases with increasing phase-matching angle (Fig. 5). As an example, $\left(\mathrm{d} \Delta k_{z} / \mathrm{d} \varphi\right)_{\max }=2460 \mathrm{~cm}^{-1}$ for the interaction (I) in fused silica, giving $\Delta k_{z, \max }=1.23 \mathrm{~cm}^{-1}$ in the case of an angular deviation of $0.5 \mathrm{mrad}$ (signal beam divergence of $\Delta \theta=1 \mathrm{mrad}$ ). In the case of collinear signal and idler light propagation (curves III in the region $\omega_{\mathrm{L}}<\omega_{\mathrm{S}}<\omega_{\mathrm{SH}}$, noncollinear pump pulse propagation) the wavevector mismatch is small.

In Fig. 19 the situation of angular spreading of one of the pump pulses is considered. In the case of collinear pump pulse propagation, the wavevector mismatch versus signal 


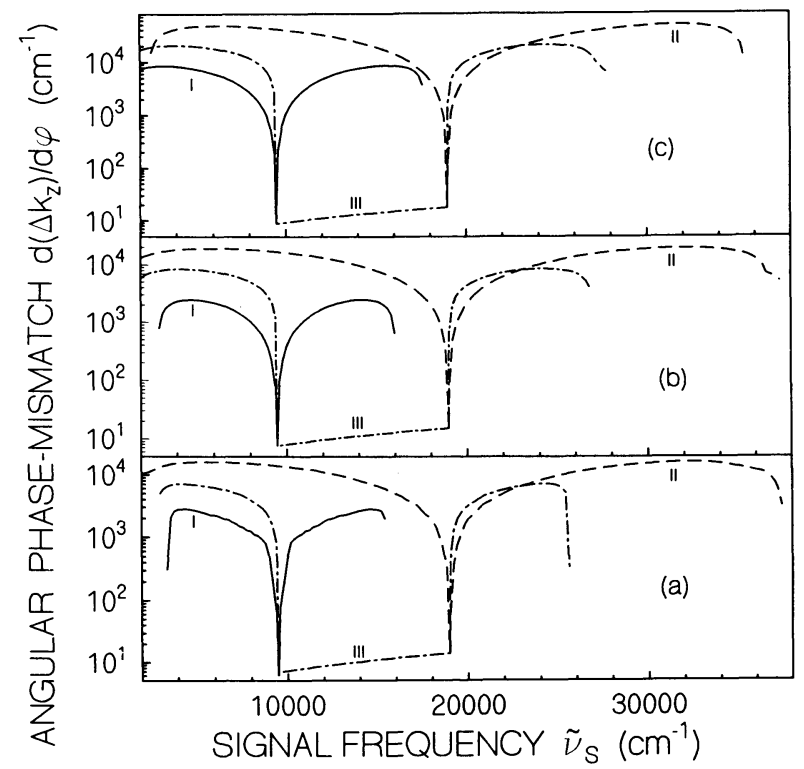

Figure 18 Wavevector-mismatch due to angular spreading of signal beam, $\mathrm{d}\left(\Delta \boldsymbol{k}_{\mathbf{z}}\right) / \mathrm{d} \varphi$, versus signal frequency $\tilde{\nu}_{S}$ for the processes (I) $\omega_{L}+\omega_{L} \rightarrow \omega_{S}+\omega_{I}$ (solid curves), (II) $\omega_{S H}+\omega_{S H} \rightarrow \omega_{S}+\omega_{I}$ (dashed

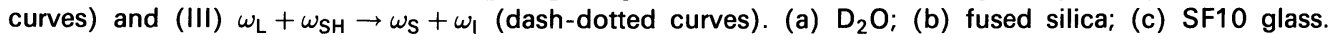
$\lambda_{\mathrm{L}}=1054 \mathrm{~nm}$. $\lambda_{\mathrm{SH}}=527 \mathrm{~nm}$.

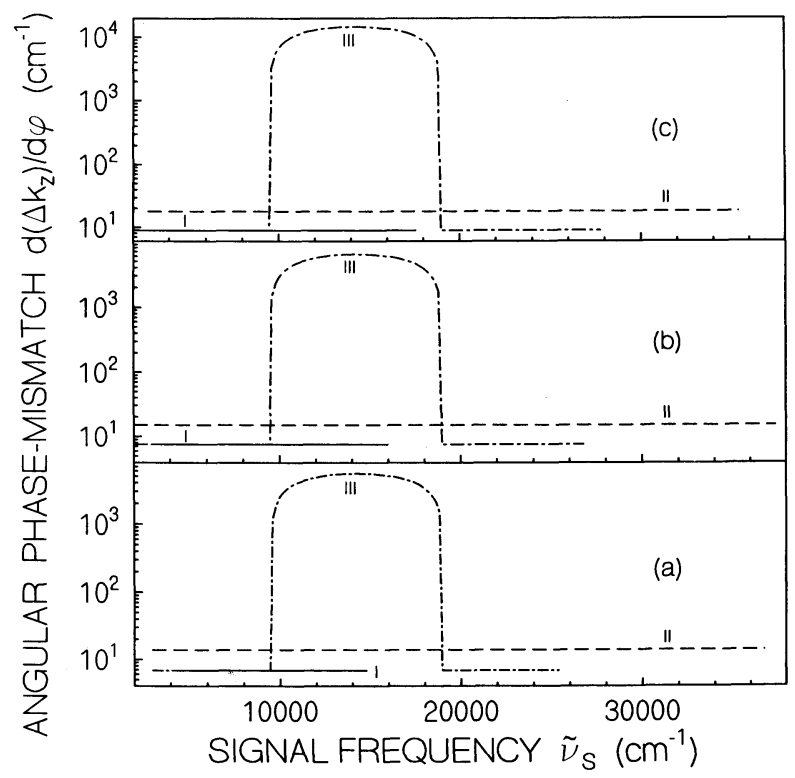

Figure 19 Wavevector mismatch due to angular spreading of one of the pump beams of angular frequency $\omega_{1}$ versus signal frequency $\tilde{\nu}_{\mathrm{S}}$ for the processes (I) $\omega_{\mathrm{L}}+\omega_{\mathrm{L}} \rightarrow \omega_{\mathrm{S}}+\omega_{\mathrm{I}}\left(\omega_{\mathrm{I}}=\omega_{\mathrm{L}}\right.$, solid curves), (II) $\omega_{\mathrm{SH}}+\omega_{\mathrm{SH}} \rightarrow$ $\omega_{S}+\omega_{I}\left(\omega_{I}=\omega_{S H}\right.$, dashed curves), and (III) $\omega_{L}+\omega_{S H} \rightarrow \omega_{S}+\omega_{I}\left(\omega_{I}=\omega_{L}\right.$, dash-dotted curves). (a) $D_{2} O$; (b) fused silica; (c) SF10 glass. $\lambda_{\mathrm{L}}=1054 \mathrm{~nm}$. $\lambda_{\mathrm{SH}}=527 \mathrm{~nm}$. 
frequency is constant and small, while in the case of noncollinear pump pulse propagation it rises with phase-matching angle $\varphi$ (curves III in the region $\omega_{\mathrm{L}}<\omega_{\mathrm{S}}<\omega_{\mathrm{SH}}$ ).

The requirement on the beam divergence $\Delta \theta$ of the signal light and the pump light is

$$
\Delta \theta \leq \frac{2 \beta_{0}}{\mathrm{~d}\left(\Delta k_{z}\right) / \mathrm{d} \varphi}
$$

in order to achieve parametric amplification over the whole angular spreading of the signal beam and to involve the pump beam over its full divergence angle in the parameteric amplification process.

\section{Comparison of materials}

The border curves $\tilde{\nu}_{\mathrm{S}, \mathrm{b}}$ of Fig. 16 show that for a fixed pulse duration $\Delta t$ and a fixed gain $G_{0}$ the covered frequency range $\delta \tilde{\nu}_{\mathrm{S}}\left(G_{0}, \Delta t\right)=\tilde{\nu}_{\mathrm{S}, \mathrm{b}, \max }\left(G_{0}, \Delta t\right)-\tilde{\nu}_{\mathrm{S}, \mathrm{b}, \min }\left(G_{0}, \Delta t\right)$ decreases in the order $\delta \tilde{\nu}_{\mathrm{S}}\left(\mathrm{SiO}_{2}\right)>\delta \tilde{\nu}_{\mathrm{S}}\left(\mathrm{D}_{2} \mathrm{O}\right)>\delta \tilde{\nu}_{\mathrm{S}}(\mathrm{SF} 10)$. $\delta \tilde{\nu}_{\mathrm{S}}\left(\mathrm{D}_{2} \mathrm{O}\right)$ is only slightly smaller than $\delta \tilde{\nu}_{\mathrm{S}}\left(\mathrm{SiO}_{2}\right)$, while $\delta \tilde{\nu}_{\mathrm{S}}(\mathrm{SF} 10)$ is rather small for $G_{0}=1$ and $\Delta t<10 \mathrm{ps}$, and for $\Delta t<1 \mathrm{~ns}$ and $G_{0}=10$. Additionally, the flint glass Schott type S59 was studied (curves obtained are not shown in this paper) and $\delta \tilde{\nu}_{\mathrm{S}}(\mathrm{SF} 59)$ was found to be slightly smaller than $\delta \nu_{\mathrm{S}}$ (SF10).

In order to compare the efficiency of parametric four-photon amplification of various media, a figure of merit

$$
f=\frac{\left|\chi_{\mathrm{P}}^{(3)}\right|^{2} I_{\mathrm{d}, \mathrm{th}}\left(\nu_{1} \nu_{2}\right)^{1 / 2} \Delta t \nu_{\mathrm{d}}}{c_{0} \epsilon_{0} \chi_{\mathrm{nl}}^{(3)^{\prime}}}
$$

may be defined by setting $f \propto \beta_{0, \max } / \Delta k_{\mathrm{SPM}, \min }$, where

$$
\beta_{0, \text { max }} / \Delta k_{\mathrm{SPM}, \text { min }} \propto\left|\chi_{\mathrm{P}}^{(3)}\right| I_{\mathrm{d}, \mathrm{th}} /\left(\kappa_{\mathrm{SPM}, z} \Delta \tilde{\nu}_{\mathrm{SPM}, \min }\right) \propto\left|\chi_{\mathrm{P}}^{(3)}\right| I_{\mathrm{d}, \mathrm{th}} \Delta t /\left(\kappa_{\mathrm{SPM}, z} \chi_{\mathrm{nl}}^{(3)} I_{\mathrm{d}, \mathrm{th}} l_{\min }\right)
$$

Using $l_{\min }=G_{0} / \beta_{0, \max } \propto\left[\left(\nu_{1} \nu_{2}\right)^{1 / 2} I_{\mathrm{d}, \mathrm{th}}\left|\chi_{\mathrm{P}}^{(3)}\right|\right]^{-1}$ and $\kappa_{\mathrm{SPM}, z} \propto \nu_{\mathrm{d}}^{-1}$ finally leads to Equation 37. $\nu_{\mathrm{d}}=\left(n_{\mathrm{d}}-1\right) /\left(n_{\mathrm{F}}-n_{\mathrm{C}}\right)$ is the Abbé number (inverse of average dispersion). $n_{\mathrm{F}}, n_{\mathrm{C}}$ and $n_{\mathrm{d}}$ are the refractive indices at $486.1 \mathrm{~nm}, 656.3 \mathrm{~nm}$ and $587.6 \mathrm{~nm}$, respectively. The figure of merit defined by Equation 37 applies as well to parametric four-photon generation and parametric four-photon oscillation.

The figures of merit $f\left(\nu_{\mathrm{L}}, \Delta t=5 \mathrm{ps}\right)$ of the investigated media are listed in Table II. They indicate that parametric four-photon amplification is most efficient for fused silica, closely followed by $\mathrm{D}_{2} \mathrm{O}$, while the efficiency is reduced for SF10 (for SF59 a figure of merit of $f \approx 0.086$ was found, similar to the $f$-value for SF10). The flint glass has the largest parametric four-photon susceptibility $\chi_{\mathrm{P}}^{(3)}$ but the self-phase modulation susceptibility $\chi_{\mathrm{nl}}^{(3)^{\prime}}$ has increased more strongly than $\chi_{\mathbf{P}}^{(3)}$ and the threshold damage intensity $I_{\mathrm{d}, \mathrm{th}}$ is lowest for this material.

Noncollinear phase-matched parametric four-photon amplification $\omega_{\mathrm{L}}+\omega_{\mathrm{L}} \rightarrow \omega_{\mathrm{S}}+\omega_{\mathrm{I}}$ $\left(\lambda_{\mathrm{L}}=1.06 \mu \mathrm{m}, \Delta t \approx 5 \mathrm{ps}\right)$ was tried in polydiacetylene crystals $\left(\chi_{\mathrm{P}}^{(3)} \approx 1.4 \times\right.$ $10^{-18} \mathrm{~m}^{2} \mathrm{~V}^{-2}$ ) [70]. In these crystals two-photon absorption attenuates the pump pulses before observation of parametric four-photon amplification (optical band gap $E_{0}<2 \hbar \omega_{\mathrm{L}}$ [71]). The figure of merit for parametric four-photon amplification in polydiacetylene crystals is expected to be rather small because $\chi_{\mathrm{nl}}^{(3)^{\prime}}$ is thought to be very large, $I_{\mathrm{d}, \text { th }}$ has to be replaced by the lower intensity $I_{\mathrm{TPA}, \text { th }}$ describing the onset intensity of 
efficient two-photon absorption $\left(I_{\text {TPA,th }} \lesssim 10^{9} \mathrm{~W} \mathrm{~cm}^{-2}[70]\right)$, and the optical dispersion is large $\left(\nu_{\mathrm{d}}\right.$ small $)$.

It should be noted that media with a large figure of merit for parametric four-photon amplification (small $\chi_{\mathrm{nl}}^{(3)^{\prime}}$ responsible for self-phase modulation, small dispersion characterized by large Abbé number, high damage threshold intensity) and low Raman gain factor are widely used for picosecond and femtosecond light continuum generation $\left(\mathrm{H}_{2} \mathrm{O}[11,12,56,72], \mathrm{D}_{2} \mathrm{O}[11,12,15,56]\right.$, mixtures of $\mathrm{H}_{2} \mathrm{O}$ and $\mathrm{D}_{2} \mathrm{O}$ [73], ethylene glycol $[74,75]$, mixtures of ethylene glycol and glycerin [76], fused silica [11, 12], optical silica fibres $[10,11,13,14,77], \mathrm{NaCl}$ crystal [12], compressed xenon gas [78, 79], compressed nitrogen gas $[78,79])$.

\section{Conclusions}

The theoretical study of noncollinear phase-matched parametric four-photon amplification presented here indicates that efficient parametric amplification is possible over wide frequency ranges for $\mathrm{D}_{2} \mathrm{O}$ and fused silica. The frequency borders are determined by wavevector mismatch due to self-phase modulation on a subpicosecond timescale and by absorption losses on a nanosecond timescale. For the flint glass SF10 (and for SF59) the self-phase modulation restricts efficient parametric four-photon amplification over wide frequency regions to nanosecond pulse durations where very high pump pulse energies would be required for reasonable parametric gain.

The noncollinear phase-matched parametric four-photon amplification may be applied to determine third-order nonlinear optical susceptibilities $\chi_{x x x x}^{(3)}\left(-\omega_{\mathrm{S}} ; \omega_{1}, \omega_{2},-\omega_{\mathrm{I}}\right)$ in various media by signal amplification measurements.

\section{References}

1. Y. R. SHEN, The Principles of Nonlinear Optics (Wiley, New York, 1984).

2. M. SCHUBERT and B. WILHELMI, Nonlinear Optics and Quantum Electronics (Wiley, New York, 1986).

3. A. LAUBEREAU, in Ultrashort Laser Pulses and Applications, edited by W. Kaiser, Topics in Applied Physics, Vol. 60 (Springer-Verlag, Berlin, 1988) p. 35.

4. V. G. DIMITRIEV, G. G. GURZADYAN and D. N. MIKOGOSYAN, Handbook of Nonlinear Optical Crystals, Springer Series in Optical Sciences, Vol. 64 (Springer-Verlag, Berlin, 1991).

5. S. A. AKHMANOV, V. A. VYSLOUKH and A. S. CHIRKIN, Optics of Femtosecond Laser Pulses (American Institute of Physics, New York, 1992) p. 137.

6. J. F. REINTJES, Nonlinear Optical Parametric Processes in Liquids and Gases (Academic Press, Orlando, 1984).

7. D. C. HANNA, A. YURATICH and D. COTTER, Nonlinear Optics of Free Atoms and Molecules (SpringerVerlag, New York, 1979).

8. S. CHEMLA and J. ZYSS, Nonlinear Optical Properties of Organic Molecules and Crystals, Vols 1 and 2 (Academic Press, Orlando, 1988).

9. P. N. PRASAD and D. J. WILLIAMS, Introduction to Nonlinear Optical Effects in Molecules and Polymers (Wiley, New York, 1991).

10. G. P. AGRAWAL, Nonlinear Fiber Optics (Academic Press, Boston, 1989).

11. R. R. ALFANO (ed.), The Supercontinuum Laser Source (Springer-Verlag, New York, 1989).

12. A. PENZKOFER and W. KAISER, Opt. Quantum Electron. 9 (1977) 315.

13. R. H. STOLEN and J. E. BJORKHOLM, IEEE J. Quantum Electron. QE-18 (1982) 1062.

14. P. WEIDNER and A. PENZKOFER, Opt. Quantum Electron. 25 (1993) 1.

15. A. PENZKOFER, A. BEIDOUN and H. J. LEHMEIER, Opt. Quantum Electron. 25 (1993) 317.

16. W. MARGULIS and U. ÖSTERBERG, Opt. Lett. 12 (1987) 519.

17. F. S. TOMKINS and R. MAHON, Opt. Lett. 6 (1981) 179. 
18. J. BOKOR, R. R. FREEMAN, R. L. PANOCK and J. C. WHITE, Opt. Lett. 6 (1981) 182.

19. L. S. GAIDA, I. S. ZEILIKOVICH and S. A. PULKIN, Opt. Spectrosc. (USSR) 64 (1988) 417.

20. L. S. GAIDA, S. A. PULKIN, I. S. ZEILIKOVICH and E. E. FRANKLIN, Opt. Spectrosc. (USSR) 65 (1988) 478.

21. V. VAICHAITIS, A. PISKARSKAS and A. UMBRASAS, Sov. J. Quantum Electron. 22 (1992) 815.

22. R. H. STOLEN, J. E. BJORKHOLM and A. ASHKIN, Appl. Phys. Lett. 24 (1974) 308.

23. K. WASHIO, K. INOUE and S. KISHIDA, Electron. Lett. 16 (1980) 658.

24. M. C. FARRIES, P. R. MORKEL and J. E. TOWNSEND, Electron. Lett. 24 (1988) 709.

25. D. C. HANNA, R. M. PERCIVAL, I. R. PERRY, R. G. SMART, P. J. SUNI, J. E. TOWNSEND and A. C. TROPPER, Electron. Lett. 24 (1988) 1111.

26. J. K. CHEE and J. M. LIU, Opt. Lett. 14 (1989) 820.

27. R. L. CARMAN, R. Y. CHIAO and P. L. KELLEY, Phys. Rev. Lett. 17 (1966) 1281.

28. D. J. HARTER, P. NARUM, M. G. RAYMER and R. W. BOYD, Phys. Rev. Lett. 46 (1981) 1192.

29. I. S. ZEILIKOVICH, S. A. PULKIN and L. S. GAIDA, Sov. Phys. JETP 60 (1984) 72.

30. I. S. ZEILIKOVICH, L. S. GAIDA, S. A. PULKIN and I. S. KOMAR, Opt. Spectrosc. 68 (1990) 385.

31. J. FRAHM and R. FISCHER, Ann. Phys. 31 (1974) 143.

32. A. VATARESCU, J. Lightwave Technol. LT-5 (1987) 1652.

33. S. J. GARTH, Appl. Opt. 31 (1992) 742.

34. S. TRILLO and S. WABNITZ, J. Opt. Soc. Am. B. 9 (1992) 1061.

35. P. N. BUTCHER and D. COTTER, The Elements of Nonlinear Optics (Cambridge University Press, Cambridge, 1991).

36. R. W. MINCK, R. W. TERHUNE and C. C. WANG, Appl. Opt. 5 (1966) 1595.

37. A. PENZKOFER, J. KRAUS and J. SPERKA, Opt. Commun. 37 (1981) 437.

38. I. GOLUB, Opt. Lett. 15 (1990) 305.

39. K. CHIKUMA and S. UMEGAKI, J. Opt. Soc. Am. B 7 (1990) 768.

40. G. I. STEGEMAN, in Contemporary Nonlinear Optics, edited by G. P. Agrawal and R. W. Boyd (Academic Press, Boston, 1992) p. 1.

41. M. BAYEN, J. Phys. Rad. 3 (1942) 57.

42. P. P. SETHNA, K. F. PALMER and D. WILliams, J. Opt. Soc. Am. 68 (1978) 815.

43. Fused quartz and fused silica for optics, Heraeus Quarz brochure 40-1015-079 (Heraeus Quarz, Buford, GA).

44. M. THALHAMMER and A. PENZKOFER, Appl. Phys. B 32 (1983) 137.

45. R. W. TERHUNE and P. D. MAKER, in Lasers, Vol. 2, edited by A. K. Levine (Marcel Dekker, New York, 1968) p. 295.

46. J. DUCUING, in Quantum Optics, edited by R. J. Glauber (Academic Press, London, 1969) p. 421.

47. A. PENZKOFER, A. LAUBEREAU and W. KAISER, Prog. Quantum Electron. 6 (1979) 55.

48. J. W. NIBLER and G. V. KNIGHTEN, in Raman Spectroscopy of Gases and Liquids, edited by A. Weber (Springer-Verlag, Berlin, 1979) p. 253.

49. S. A. J. DRUET and J. P. E. TARAN, Prog. Quantum Electron. 7 (1981) 1.

50. W. M. TOLLES, J. W. NIBLER, J. R. McDONALD and A. B. HARVEY, Appl. Spectrosc. 31 (1977) 253.

51. A. PENZKOFER and W. LEUPACHER, Opt. Quantum Electron. 19 (1988) 327.

52. J. C. DIELS, in Dye Laser Principles with Applications, edited by F. J. Duarte and L. W. Hillman (Academic Press, Boston, 1990) p. 41.

53. Y. R. SHEN and G.-Z. YANG, in The Supercontinuum Laser Source, edited by R. R. Alfano (Springer-Verlag, New York, 1989) p. 1.

54. P. L. BALDECK, P. P. HO and R. R. ALFANO, in The Supercontinuum Laser Source, edited by R. R. Alfano (Springer-Verlag, New York, 1989) p. 117.

55. N. BLOEMBERGEN, IEEE J. Quantum Electron. QE-10 (1974) 375.

56. W. L. SMITH, P. LIU and N. BlOEMBERGEN, Phys. Rev. A 15 (1977) 2396.

57. H. HACK and N. NEUROTH, Appl. Opt. 21 (1982) 3239.

58. W. KOECHNER, Solid-State Laser Engineering, 2nd edn (Springer-Verlag, Berlin, 1988) p. 549.

59. A. J. GLASS and A. H. GUENTHER, Appl. Opt. 18 (1978) 2112.

60. A. PENZKOFER and W. LEUPACHER, Opt. Quantum Electron. 20 (1988) 227.

61. O. SVELTO, Prog. Opt. 12 (1974) 1.

62. B. MEIER, P. WEIDNER and A. PENZKOFER, Appl. Phys. B 51 (1990) 404.

63. A. PENZKOFER, Opt. Quantum Electron. 23 (1991) 685.

64. R. H. STOLEN, Proc. IEEE 68 (1980) 1232. 
65. S. S. BALLARD, J. S. BROWDER and J. F. EBERSOLE, in American Institute of Physics Handbook, 3rd edn, edited by D. E. Gray (McGraw-Hill, New York, 1972) p. 69.

66. Optical Glass, technical information (Schott Glaswerke, Mainz, 1992).

67. R. H. STOLEN and C. LIN, Phys. Rev. A 17 (1978) 1448.

68. O. RAHN, M. MAIER and W. KAISER, Opt. Commun. 1 (1969) 109.

69. D. HEIMAN, R. W. HELLWARTH and D. S. HAMILTON, J. Non-Cryst. Solids 34 (1979) 63.

70. J. P. HERMANN and M. LEQUIME, in Picosecond Phenomena, edited by C. V. Shank, E. P. Ippen and S. L. Shapiro, Springer Series in Chemical Physics, Vol. 4 (Springer-Verlag, Berlin, 1978) p. 40.

71. C. FLYTZANIS, in Nonlinear Optical Properties of Organic and Polymeric Materials, edited by D. J. Williams, ACS Symposium Series, Vol. 233 (American Chemical Society, Washington, D.C., 1983) p. 167.

72. A. MiguS, A. ANTONETTI, J. ETCHEPARE, D. HUlin and A. ORZAG, J. Opt. Soc. Am. B 2 (1985) 584.

73. M. HYDE and G. S. BEDDARD, Chem. Phys. 151 (1991) 239.

74. R. L. FORK, C. V. SHANK, C. A. HIRLIMANN and W. J. TOMLINSON, Opt. Lett. 8 (1983) 1.

75. W. H. KNOW, M. C. DOWNER, R. L. FORK and C. V. SHANK, Opt. Lett. 9 (1984) 552.

76. G. R. OlBRight and G. R. HADLEY, J. Opt. Soc. Am. B 6 (1969) 1363.

77. P. L. BALDECK, P. P. HO and R. R. AlfANO, Rev. Phys. Appl. 22 (1987) 1877.

78. P. B. CORKUM and C. ROLLAND, Phys. Rev. Lett. 57 (1986) 2268.

79. P. B. CORKUM and C. ROLLAND, IEEE J. Quantum Electron. 25 (1989) 2634.

80. R. W. MINCK, R. W. TERHUNE and C. C. WANG, Appl. Opt. 5 (1966) 1595. 